\title{
Dehn Surgeries on Knots Creating Essential Tori, II
}

\author{
C. McA. Gordon ${ }^{1}$ AND J. LUECKE ${ }^{2}$
}

\section{Introduction.}

In this paper, which is a sequel to [GLu1], we continue our study of when Dehn surgery on a hyperbolic knot $K$ in $S^{3}$ can yield a manifold that contains an incompressible torus.

Let $E(K)$ denote the exterior of $K$, and let $K(\gamma)=E(K) \cup V_{\gamma}$ be the closed 3-manifold obtained by $\gamma$-Dehn surgery on $K$; thus $V_{\gamma}$ is a solid torus whose meridian is identified with the slope $\gamma$ on $\partial E(K)$. Suppose that $K(\gamma)$ contains an incompressible torus $\widehat{T}$. We assume that $K_{\gamma}$, the core of $V_{\gamma}$, intersects $\widehat{T}$ transversely and that $\widehat{T}$ is chosen (among all incompressible tori in $K(\gamma))$ to minimize $t=\left|\widehat{T} \cap K_{\gamma}\right|$.

Let $\mu$ be the meridian of $K$.

In [GLu1] we showed that $\Delta(\gamma, \mu)$, the minimal geometric intersection number of $\gamma$ and $\mu$ on $\partial E(K)$, is at most 2, and that if $\Delta(\gamma, \mu)=2$, then $t=2$ or 4 . In the present paper we eliminate the case $t=4$. This completes the proof of [GLu1,Theorem 1.2], which we restate here for the reader's convenience. ( $T$ denotes the punctured torus $\widehat{T} \cap E(K)$ ).

Theorem. Suppose that $K(\gamma)$ contains an incompressible torus, where $\Delta(\gamma, \mu)=2$. Then $t=2$, and $T$ separates $E(K)$ into two genus 2 handlebodies. In particular, $K$ is strongly invertible. Furthermore, the tunnel number of $K$ is at most 2.

As mentioned in [GLu1], infinitely many examples of such knots have been described by Eudave-Muñoz [EM2].

We assume familiarity with [GLu1]. In particular, recall the graphs $G_{Q}$, $G_{T}$ in $\widehat{Q}$ and $\widehat{T}$, where $\widehat{Q}$ is a suitable level 2-sphere in $S^{3}$, defined by the arcs of intersection of $Q=\widehat{Q} \cap E(K)$ and $T$. By [GLu1, Corollary 2.7], $G_{Q}$

\footnotetext{
${ }^{1}$ Partially supported by NSF Grant DMS-9303229

${ }^{2}$ Partially supported by an NSF Presidential Young Investigator Award and Texas Advanced Research Project Award
} 
contains a great web $\Lambda$ (see [GLu1,Section 2] for definitions). We assume that $\Delta(\gamma, \mu)=2$ and $t=4$, and eventually obtain a contradiction. Roughly speaking, this is achieved by showing that, on the one hand, using Euler characteristic arguments, $\Lambda$ must contain certain configurations of faces of length $\leq 4$, while on the other hand these configurations are impossible for various topological reasons. We remark that one of the lines of argument we use is to show that certain configurations of faces imply that $K$ is strongly invertible, in which case $t=2$ by a result of Eudave-Muñoz [EM1].

Here is a more detailed description of the argument and the organization of the paper.

Recall from [GLu1] that for each label $x$ of $G_{Q}$ (here $\left.x \in\{1,2,3,4\}\right), \Lambda_{x}$ is the subgraph of $\Lambda$ whose vertices are the vertices of $\Lambda$ and whose edges are the $x$-edges in $\Lambda$. Since all the vertices of $\Lambda$ have the same sign, each edge of $\Lambda_{x}$ has exactly one end with label $x$, by the parity rule.

For clarity of exposition, the proof is given in the early Sections 2, 3 and 4, while the technical results on which the arguments of these sections depend are postponed until Sections 5, 6 and 7. Sections 5 and 6 are devoted to ruling out various configurations in $G_{Q}$, with Section 6 being reserved for those arguments that involve strong invertibility. Section 7 uses Euler characteristic arguments on the graphs $\Lambda_{x}$ and $\Lambda$ to show that (for each label $x$ ) $\Lambda_{x}$ must contain certain faces of lengths 2,3 and 4 , and also that $\Lambda$ must contain a "special" vertex to which certain faces of lengths 2,3 and 4 are incident.

In Section 2 we show, by analyzing the faces of $\Lambda_{x}$ of lengths 2 and 3 , that $G_{Q}$ must contain certain Scharlemann cycles (see e.g., [GLu1] for definition) of lengths 2 and 3 . This is done by showing that, firstly, by an easy Euler characteristic argument, each $\Lambda_{x}$ must contain a face of length 2 or 3 , and secondly, by results from Sections 5 and 6 , any such face must be a Scharlemann cycle. In Section 3 we extend the result of Section 2 to conclude that $G_{Q}$ actually contains a Scharlemann cycle of length 2, 3 or 4 on each of the four label-pairs of $G_{Q}$. The proof here follows the same philosophy as in Section 2, but is considerably more difficult. Again an Euler characteristic argument (Section 7) gives a lower bound on the number of faces of $\Lambda_{x}$ of lengths 2, 3 and 4, and again most such faces are eliminated by results from Sections 5 and 6 . For the remainder, we show that the possibilities for two such faces to share a vertex are sufficiently restricted that, unless the desired Scharlemann cycles exist, the lower bound mentioned above simply gives rise to too many vertices in $\Lambda$. The whole argument is completed in Section 4 , which shows that each of the three possibilities for the Scharlemann cycles, listed in Theorem 3.1, leads to a contradiction. This is done by showing 
that the special vertex of $\Lambda$ mentioned above yields faces of $G_{Q}$ which are incompatible (again by the results of Section 5 and 6) with the Scharlemann cycles in question.

We conclude this introduction by describing some terminology that will be used throughout the paper.

We will denote the four labels of $G_{Q}$ (vertices of $G_{T}$ ) by $a, b, c, d$. Thus $(a, b, c, d)$ stands for any of the ordered 4-tuples $(1,2,3,4),(2,3,4,1),(3,4,1,2)$, $(4,1,2,3)$.

A face of $G_{Q}$ either has $a b$ - and $c d$-corners, or $b c$ - and $d a$-corners. A face of $G_{Q}$ whose vertices all have the same sign (as will be the case for faces of $\Lambda$ ), and which has at least one $a b$-corner and at least one $c d$-corner will be called an $(a b, c d)$-face. In particular, we shall refer to $(a b, c d)$-bigons and $(a b, c d)$-3-gons.

By an $a b$-edge we will mean either an edge of $G_{T}$ joining vertices $a$ and $b$, or the corresponding edge of $G_{Q}$ with labels $a$ and $b$ at its endpoints. $\left.G_{T}\right\}$.

The edge class of an edge of $G_{T}$ is its isotopy class in $\widehat{T}$ rel\{vertices of

We are grateful to Masakazu Teragaito for pointing out an error in the original manuscript.

\section{The 3-gons of $\Lambda_{x}$.}

The goal of this section is to prove Corollary 2.4, which asserts that $G_{Q}$ must contain certain Scharlemann cycles of lengths 2 and 3.

Theorem 2.1. For every label $x, \Lambda_{x}$ contains a face of length 2 or 3 .

Proof. This follows from an Euler characteristic argument and is done in Theorem 5.5 of [GLu1]. In fact, the argument is easier here since by Theorem 5.6 of [GLu1], $\alpha_{x} \leq 2$.

Figure 2.1 lists all possible faces of $\Lambda_{x}$ of length at most 3 , when $x=4$. (By abuse of terminology, when we talk about a face $f$ of $\Lambda_{x}$, we shall frequently mean the subgraph of $\Lambda$ consisting of all the edges of $\Lambda$ contained in $f$.)

Theorem 2.2. For every label $x, \Lambda$ contains a Scharlemann cycle of length at most 3 with $x$ as a label. 
674

Cameron Gordon and John Luecke
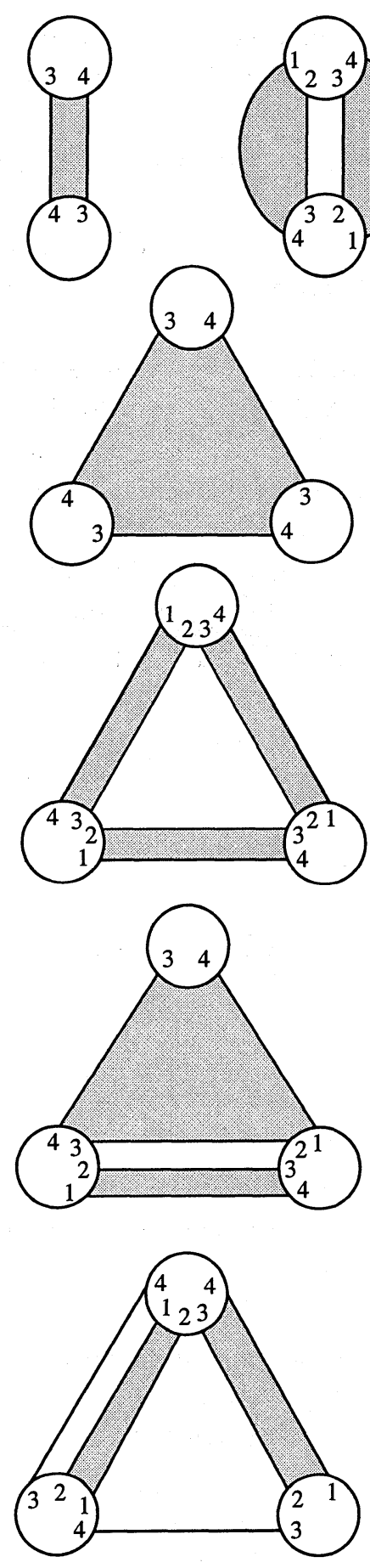
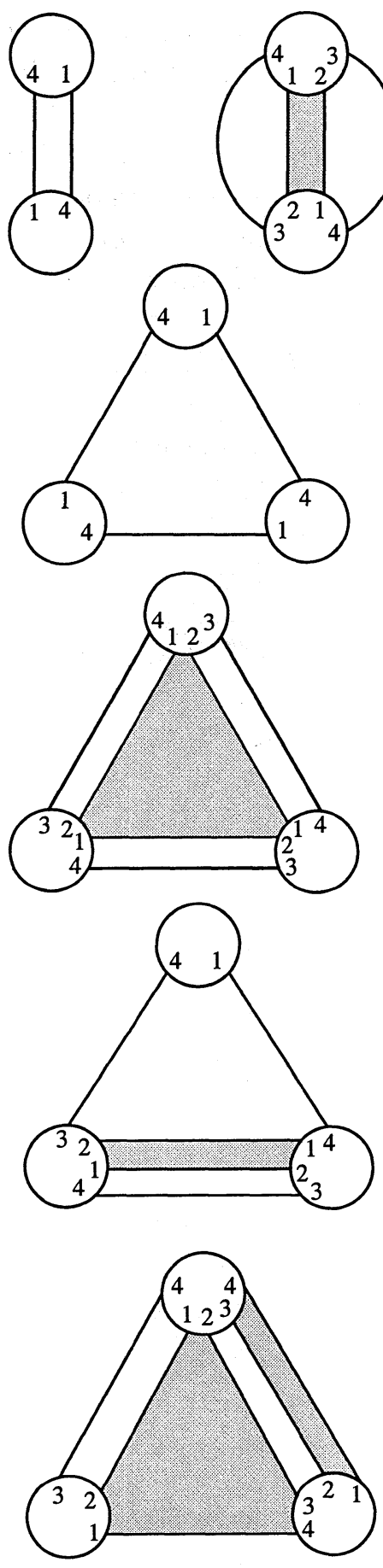

Figure 2.1. 


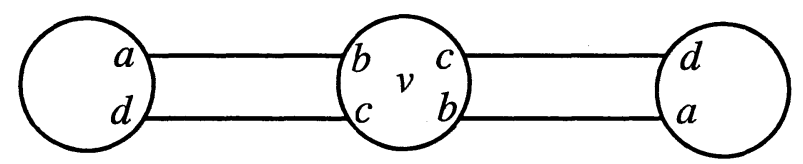

Figure 2.2.

Proof. Without loss of generality assume $x=4$. Then $\Lambda_{4}$ has a face $f$ of length at most 3 . Figure 2.1 shows all possibilities for $f$. But Theorems 5.1 and 6.3 rule out all configurations except the Scharlemann cycles.

Theorem 2.3. $\Lambda$ contains a Scharlemann cycle of length 2 .

Proof. If not then Theorem 2.2 guarantees that there are two Scharlemann cycles of length 3 on disjoint label-pairs $a b$ and $c d$. By Corollary 7.3 there is a vertex $v$ of $\Lambda$ to which three bigons of $\Lambda$ are incident. Theorem 5.12 along with the assumption that there are no Scharlemann cycles of length 2 imply that each of these three bigons is a $(b c, d a)$-bigon. Hence there are two such bigons incident to $v$ at the same label-pair; see Figure 2.2. But this contradicts Corollary 5.4.

Corollary 2.4. After possibly relabelling, $G_{Q}$ contains a 12-Scharlemann cycle of length 2, and either

(A) a 34-Scharlemann cycle of length 3; or

(B) a 23-Scharlemann cycle of length 3 and a 41-Scharlemann cycle of length 3 .

Proof. By Theorem 2.3 we may relabel so that there is a 12-Scharlemann cycle of length 2. Applying Theorem 2.2 with $x=3$ and 4 implies that there is either a 23-Scharlemann cycle of length 2 or 3 or a 34-Scharlemann cycle of length 2 or 3 , and either a 41-Scharlemann cycle of length 2 or 3 or a 34-Scharlemann cycle of length 2 or 3 . By Theorem 5.10, there can be no 34-Scharlemann cycle of length 2. Hence if Case A does not occur then there are 23- and 41-Scharlemann cycles of length at most 3. Therefore, again using Theorem 5.10, either Case B occurs or by relabelling we are in Case A. 


\section{The 4-gons of $\Lambda_{x}$.}

The goal of the present section is to prove

Theorem 3.1. For each label $x, G_{Q}$ contains an $x, x+1-S c h a r l e m a n n$ cycle, $\sigma_{x}$, of length at most 4 . In particular, if $m_{x}$ denotes the length of $\sigma_{x}$ then we may assume that $\left(m_{1}, m_{2}, m_{3}, m_{4}\right)$ is one of the following: $(2,2,3,3)$, $(2,3,3,3),(2,3,3,4)$.

After Corollary 2.4, we assume throughout this section that $G_{Q}$ contains a 12-Scharlemann cycle of length 2 .

We analyze the faces of $\Lambda_{4}$ of length 4 and show that only a small number of types of such faces can exist. This argument splits into Cases A and B of Corollary 2.4. An Euler characteristic argument then shows that in Case A there must be a 41-Scharlemann cycle of length at most 4, while in Case B there must be a 34-Scharlemann cycle of length 3 . In Case A, we apply the same argument to $\Lambda_{3}$ to show that there must also be a 23-Scharlemann cycle of length at most 4 . The proof of Theorem 3.1 appears at the end of the section.

The possible faces of $\Lambda_{4}$ of length 4 are listed in Figure 3.1.

Theorem 3.2. Only configurations 7, 8, 14 and 20 of Figure 3.1 may appear in $G_{Q}$.

Proof. Theorem 6.3 rules out configurations 4, 10, 15, 16, 21, 22, 23 and 24.

Theorem 5.1 rules out configurations 6 and 12 .

Theorem 5.10 rules out configuration 1.

Theorem 5.18 rules out configuration 2 .

Theorem 5.16 rules out configurations 5 and 11 .

The argument now splits into Cases A and B of Corollary 2.4.

Case A. $G_{Q}$ contains a 34-Scharlemann cycle of length 3 .

Theorem 5.14 now rules out configurations $3,13,17$ and 18 .

Theorem 5.21 rules out configurations 9 and 25 .

Theorem 6.14 rules out configuration 19 .

Theorem 6.9 rules out configuration 26. In this case we take $a, b, c, d$ to be $2,1,4,3$ (and apply an orientation-reversing homeomorphism to $\widehat{Q}$, to conform to our convention that the labels appear in anticlockwise order). The hypothesis of Theorem 6.9 that there are $b c$-edges which are not parallel 
Faces of $\Lambda_{4}$ of length 4
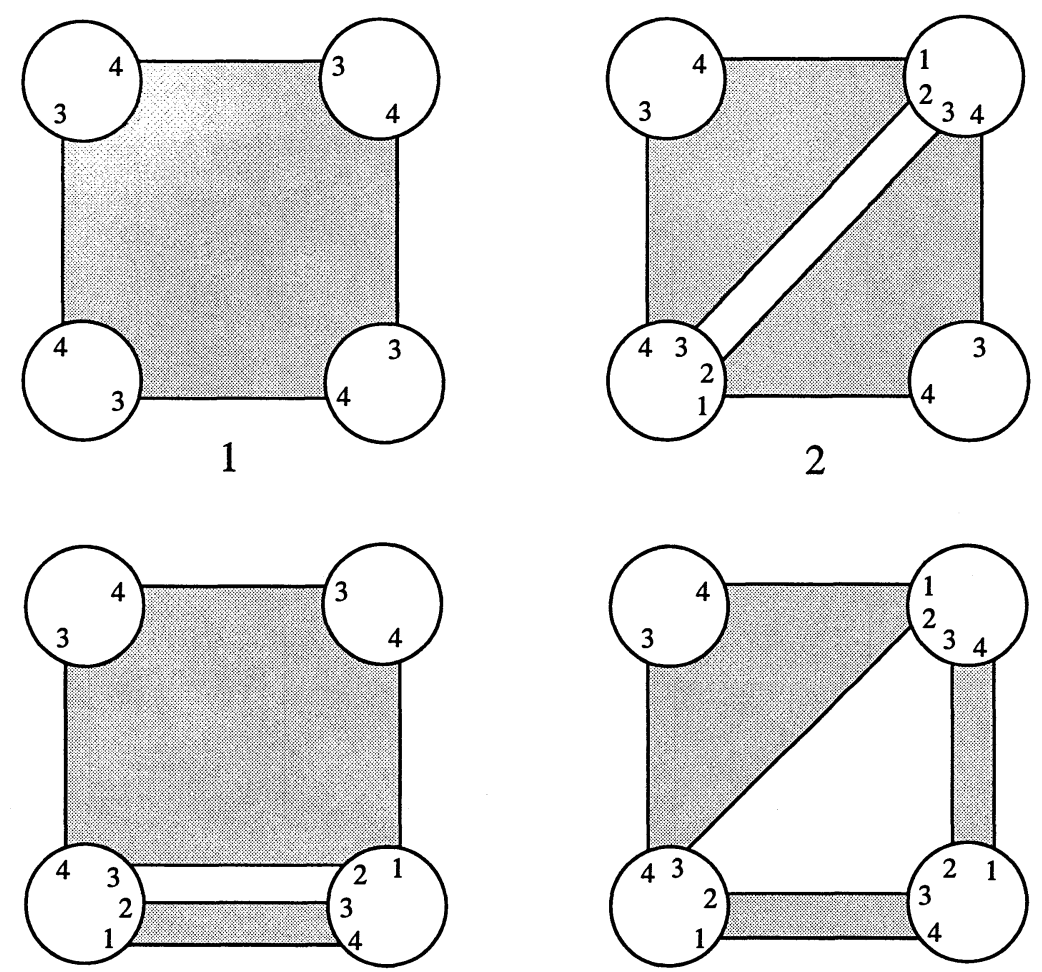

3

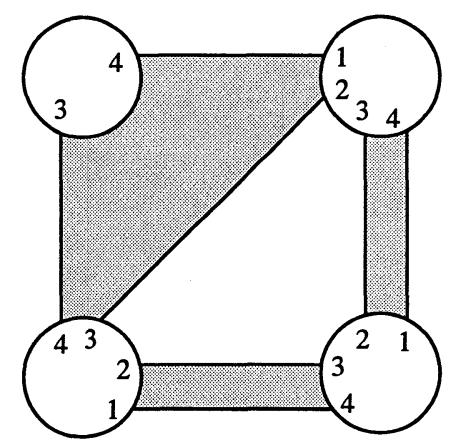

4
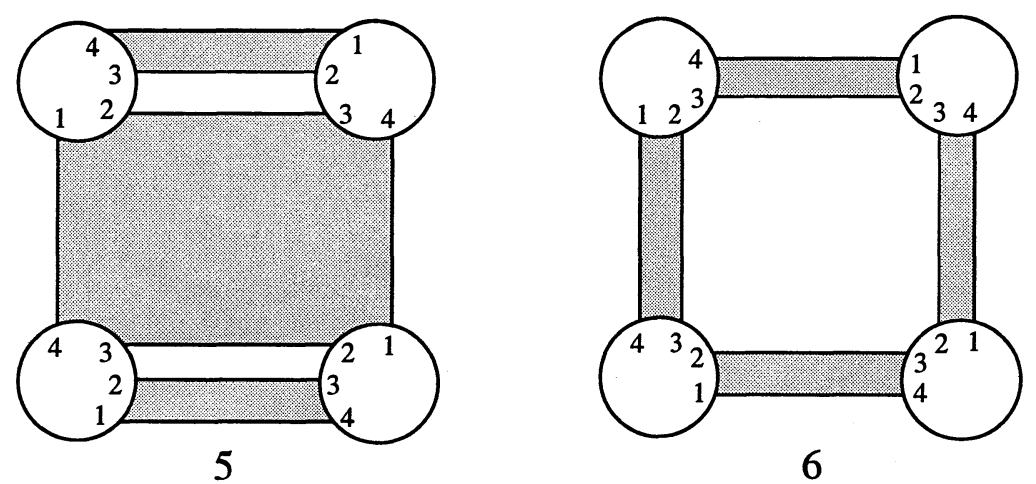

Figure 3.1. 

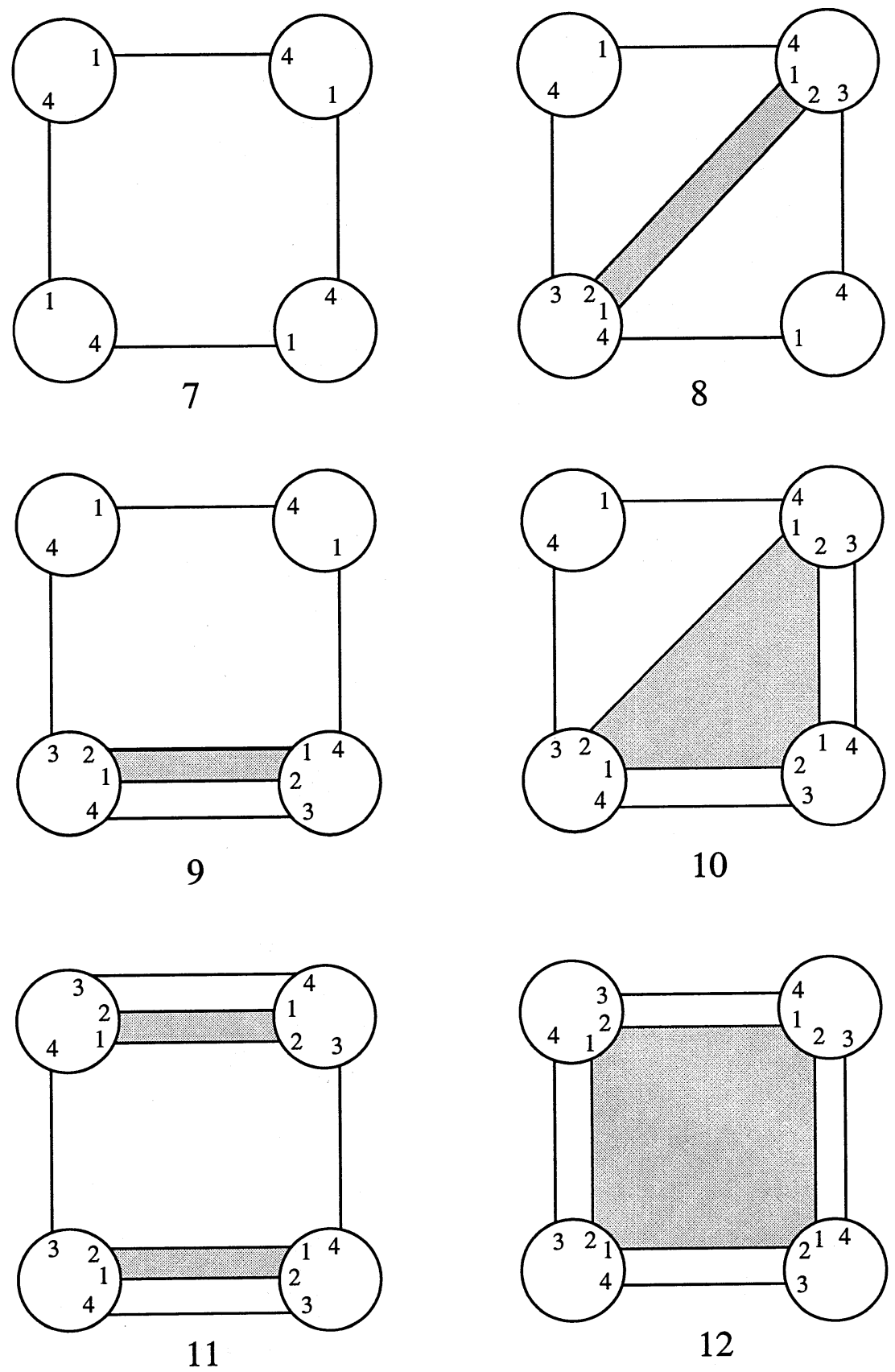

Figure 3.1. 

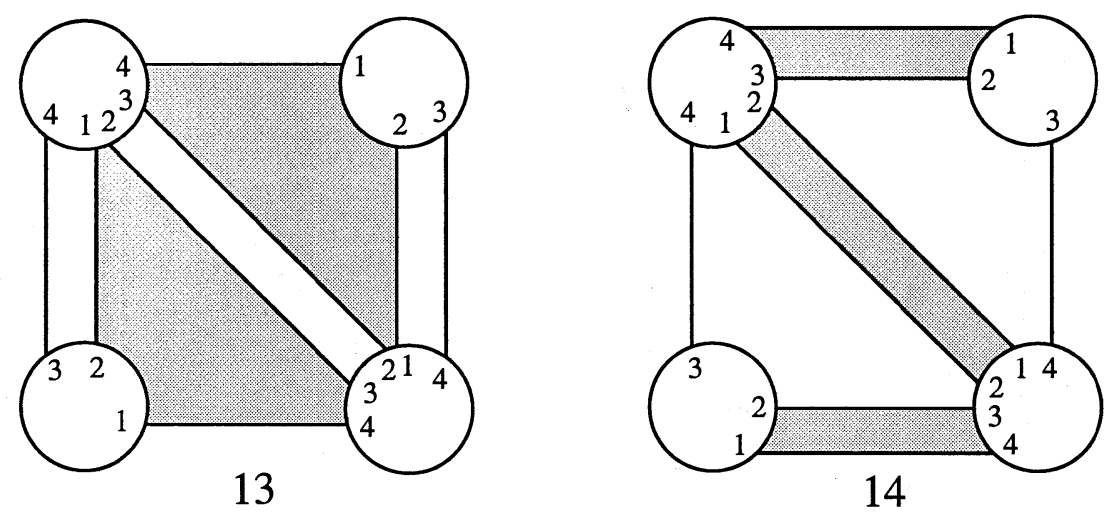

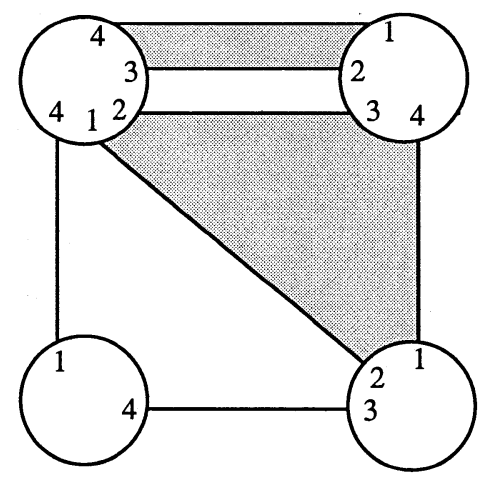

15

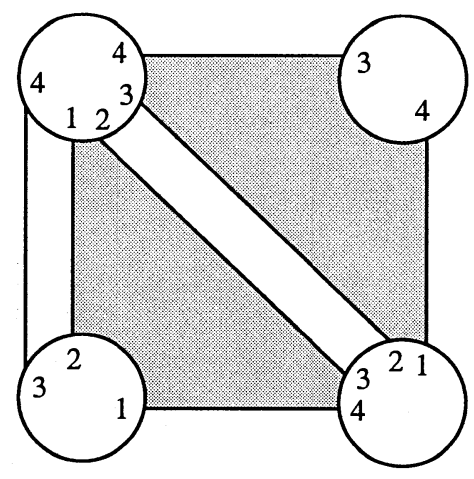

17

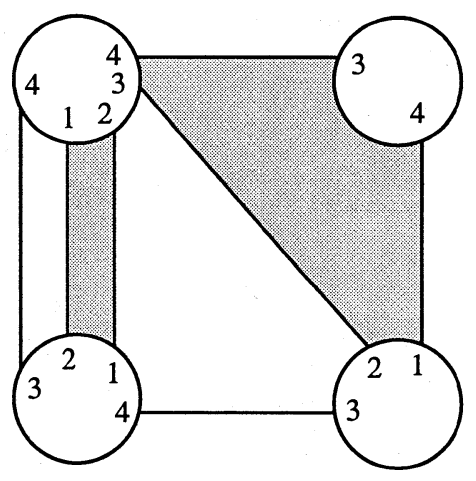

16

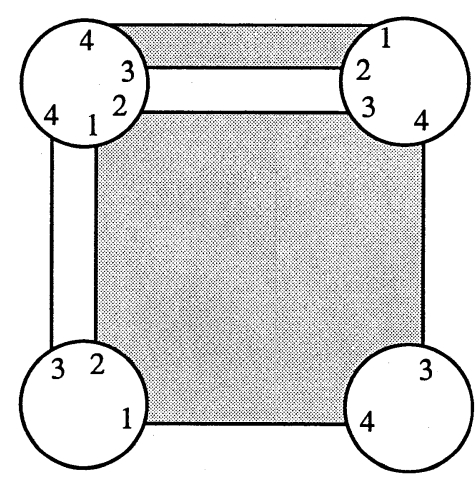

18

Figure 3.1. 

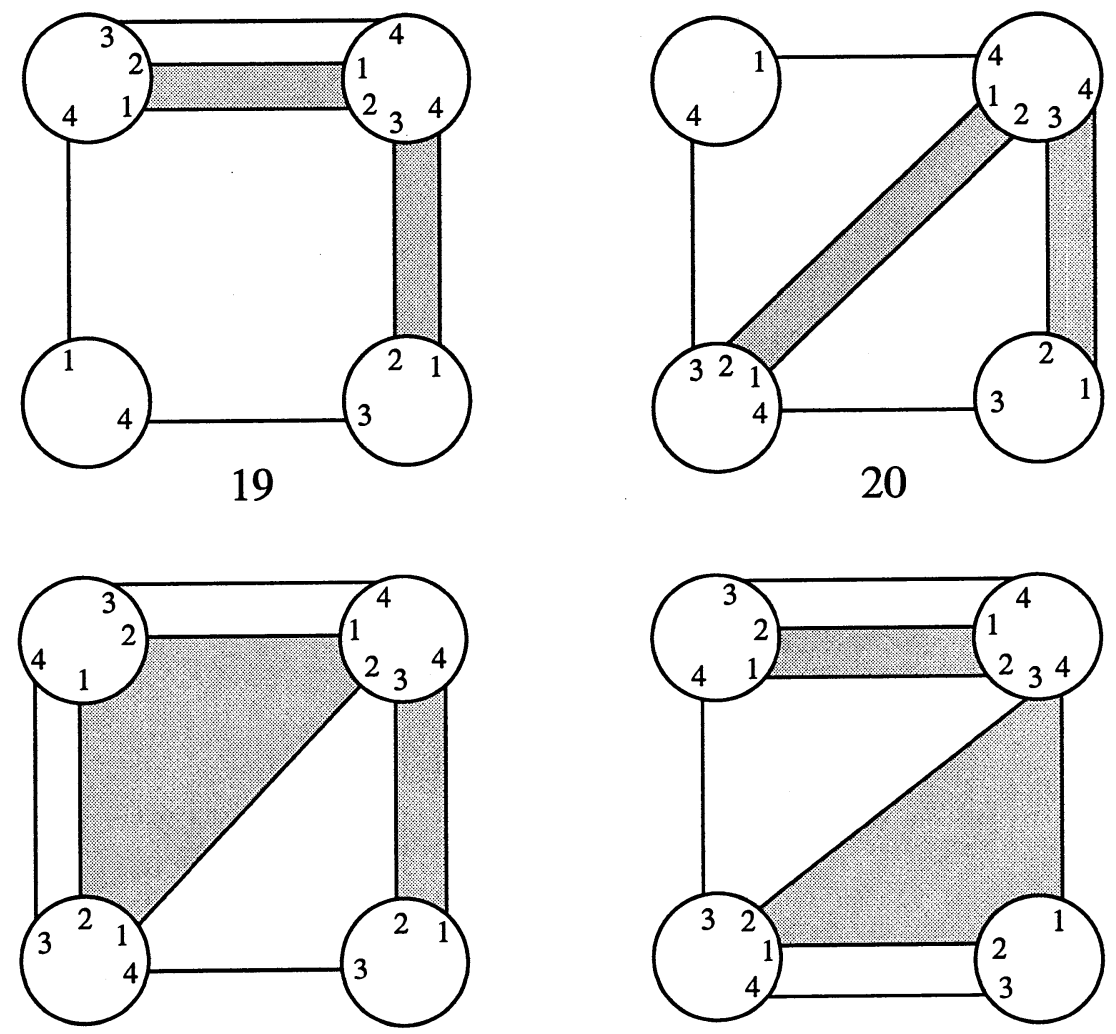

21
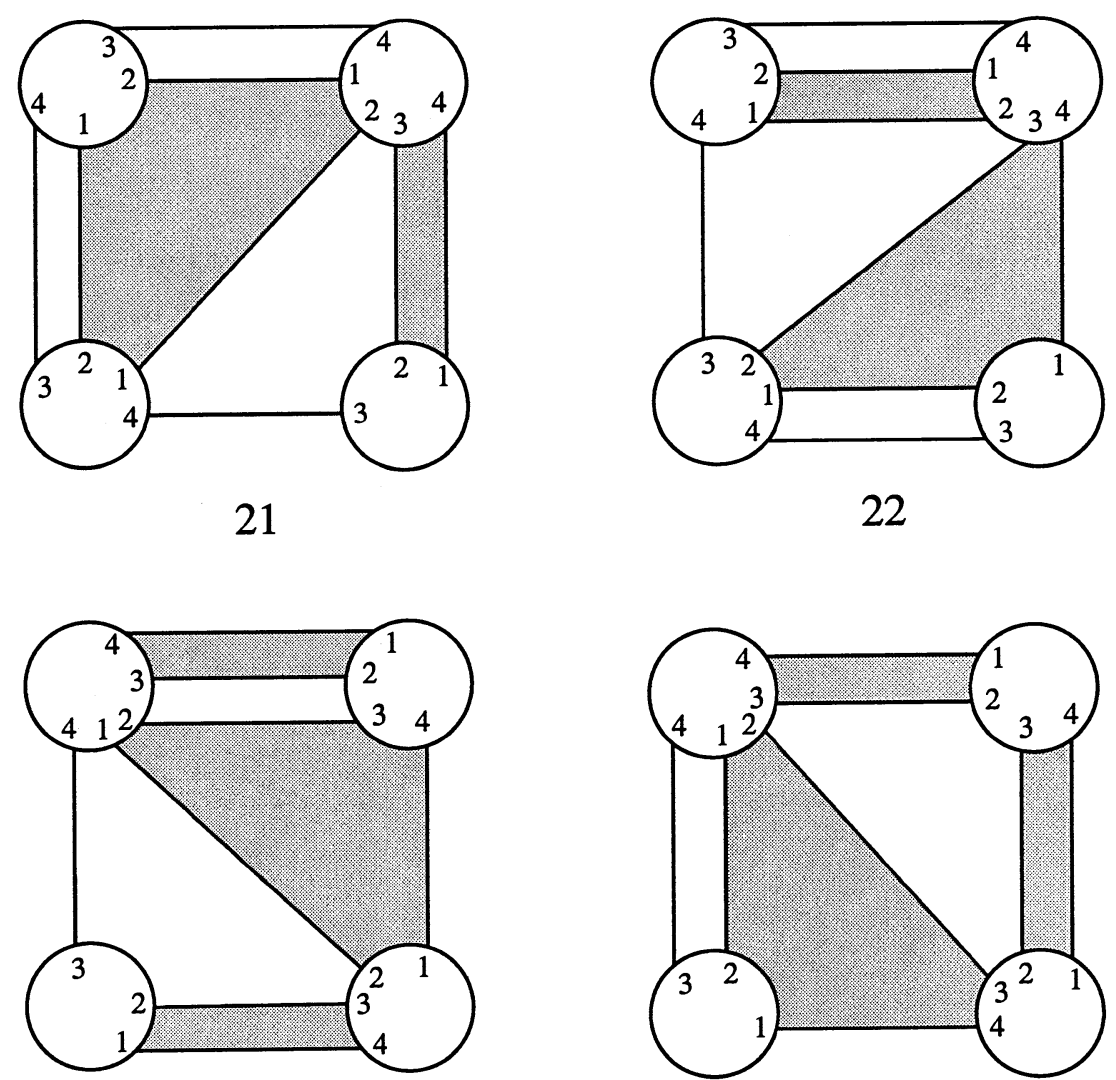

23

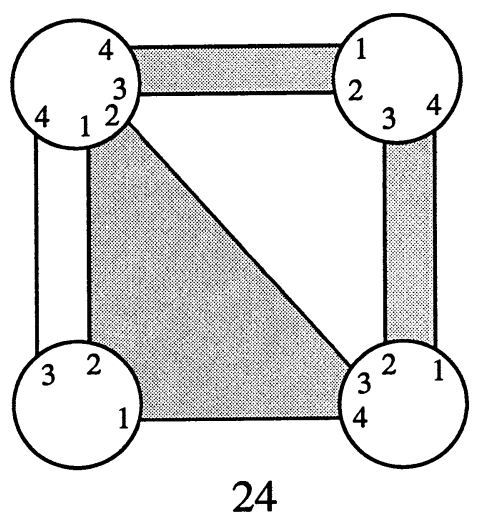

Figure 3.1. 


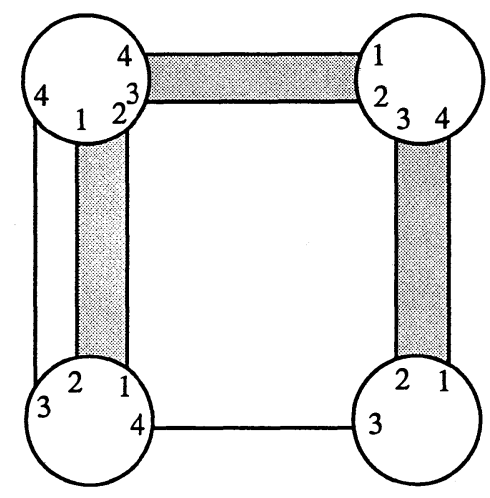

25

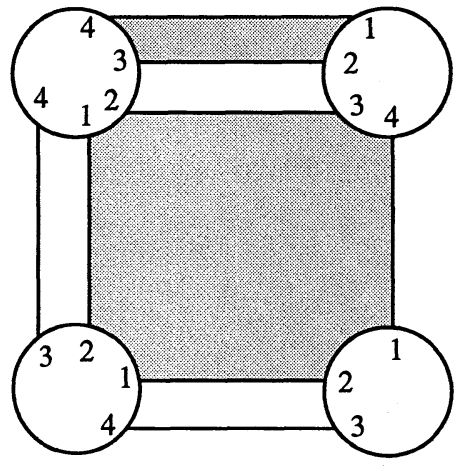

26

Figure 3.1.

is not clear in this context. However, we can supplant this hypothesis with the fact that the $d a$-Scharlemann cycle shares an edge with the $(a b, c d)$ bigon. In particular, we use this fact to guarantee, just after the proof of Claim 6.10, that edges of faces in $G_{Q}$ under consideration appear in $G_{T}$ as in Figure 6.12.

This completes the proof of Theorem 3.2 in Case A.

Case B. $G_{Q}$ contains 23- and 41-Scharlemann cycles of length 3.

Theorem 5.12 rules out configurations $9,13,17,18,19,25$ and 26 .

Theorem 5.7 rules out configuration 3 .

This completes the proof of Theorem 3.2 in Case B.

Theorem 3.3. In Case B of Corollary $2.4 G_{Q}$ contains a 34-Scharlemann cycle of length 3 .

Proof. Assume not for contradiction.

By Theorem 5.10, $G_{Q}$ contains no 34-Scharlemann cycle of length 2 (or 4). By Theorem 5.7, $G_{Q}$ contains no 41-Scharlemann cycle of length 2 or 4 . As argued in the proof of Theorem 2.2, any face of $\Lambda_{4}$ of length at most 3 is a Scharlemann cycle (with 4 as a label). Hence any face of $\Lambda_{4}$ of length at most 3 is a $41-$ Scharlemann cycle of length 3 . 

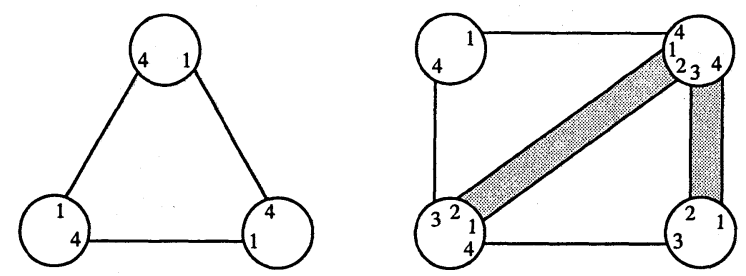

Figure 3.2.

Now consider the faces of $\Lambda_{4}$ of length 4 listed in Theorem 3.2. As noted above, configuration 7 does not occur. Furthermore, Theorem 5.18 rules out configurations 8 and 14 . We conclude that the only faces of $\Lambda_{4}$ of length at most 4 are the two pictured in Figure 3.2.

Let I and II be the 41-edge classes given by Theorem 5.8. By Theorem 5.9, the 41-edge in the 3-gon face of configuration 20 is in class II. To each 41-Scharlemann cycle of length 3 in $\Lambda_{4}$ assign its two 41-edges in class II, and to each face of $\Lambda_{4}$ as in configuration 20 assign the 41-edge in class II just described. Note that under this rule the same edge is never assigned twice. Hence if $n_{k}^{4}$ is the number of faces of $\Lambda_{4}$ of length $k$, the number of 41-edges of $\Lambda_{4}$ in class II is at least $2 n_{3}^{4}+n_{4}^{4}$. Since $n_{2}^{4}=0$, and since $3 n_{2}^{4}+2 n_{3}^{4}+n_{4}^{4}>V$ by Theorem 7.1, where $V$ is the number of vertices of $\Lambda_{4}$, we conclude that the number of 41-edges of $\Lambda_{4}$ in class II is greater than $V$. Hence there is a vertex of $\Lambda_{4}$ at which two 41-edges in class II are incident with label 4. But this contradicts Theorem 5.2.

This contradiction shows that there must be a 34-Scharlemann cycle of length 3 , proving the theorem.

Theorem 3.4. In Case $A$ of Corollary $2.4 G_{Q}$ contains a 41-Scharlemann cycle of length at most 4 .

Proof. Assume not for contradiction. Then, as argued in Theorem 2.2, any face of $\Lambda_{4}$ of length at most 3 is a 34-Scharlemann cycle. Note also that by Theorem 5.10 (or Theorem 5.7) there can be no 34-Scharlemann cycle of length 2 . Combining this with Theorem 3.2 , we have that the only possible faces of $\Lambda_{4}$ of length at most 4 are 34-Scharlemann cycles of length 3 together with configurations 8, 14 and 20 of Figure 3.1. By Theorem 6.11, no two of the configurations 8,14 and 20 may appear together. Thus there are three possibilities for the faces of $\Lambda_{4}$ of length at most 4:

(1) 34-Scharlemann cycle, 8 


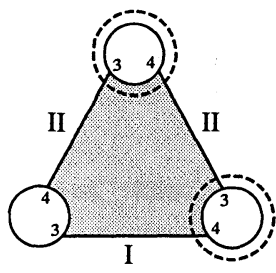

(i)

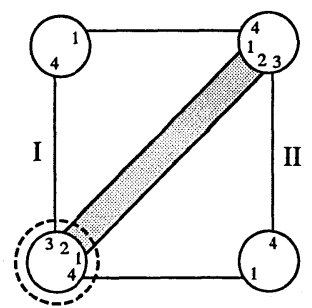

(ii)

Figure 3.3.

(2) 34-Scharlemann cycle, 14

(3) 34-Scharlemann cycle, 20

As in the proof of Theorem 3.3, Theorem 7.1 implies that

$$
2 n_{3}^{4}+n_{4}^{4}>V
$$

In each of the three cases above we will use this to arrive at a contradiction.

Case (1). Let I and II be the 34-edge classes given by Theorem 5.8. Note that the 12-Scharlemann cycle forces any 34-edge to be in class I or II. Hence by Theorem 5.17, in any occurrence of configuration 8, one of the 34-edges is in class I and the other is in class II. To each 34-Scharlemann cycle in $\Lambda_{4}$, assign the two vertices shown in Figure 3.3(i), and to each face of $\Lambda_{4}$ as in configuration 8, assign the vertex shown in Figure 3.3(ii).

By the inequality (3.1), some vertex is assigned twice, i.e., there must be some vertex at which two distinct corners in faces of $\Lambda_{4}$ as in Figure 3.3 are incident. But this would produce two 34-edges in the same class incident to this vertex with label 3 , contradicting Theorem 5.2.

Case (2). To the faces of $\Lambda_{4}$ of length at most 4 we assign the vertices shown in Figure 3.4 (again Theorem 5.17 guarantees that in configuration 14 one 34-edge is in class I and the other is in class II).

By (3.1) there is a vertex of $\Lambda_{4}$ at which two distinct corners in faces as in Figure 3.4 are incident. But this would produce two 34-edges in class II incident to this vertex with label 4, again contradicting Theorem 5.2.

Case (3). Here we assign the vertices shown in Figure 3.5.

By (3.1) there is a vertex $v$ of $\Lambda_{4}$ at which two corners of these faces are incident. By Theorem 5.2, the only possibility is that both faces are configuration 20. See Figure 3.6. 

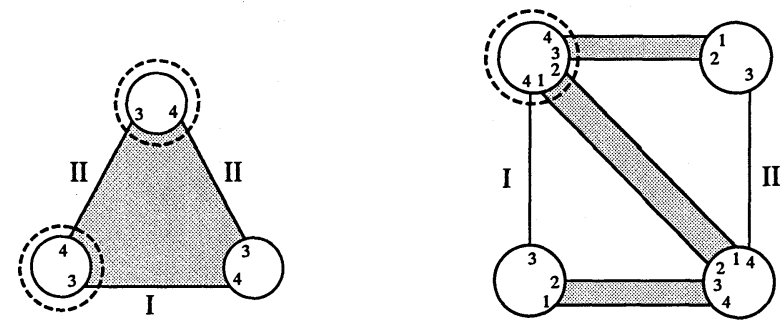

Figure 3.4.
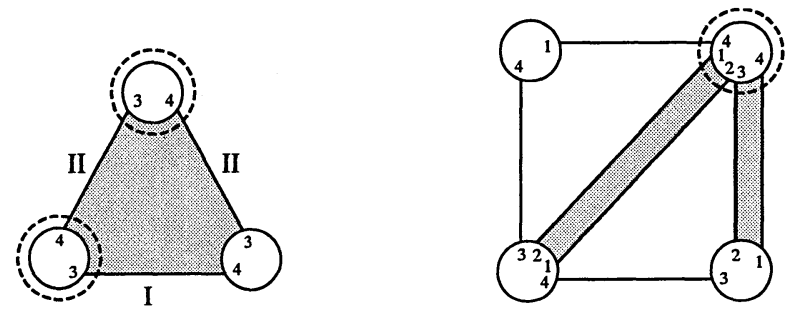

Figure 3.5.

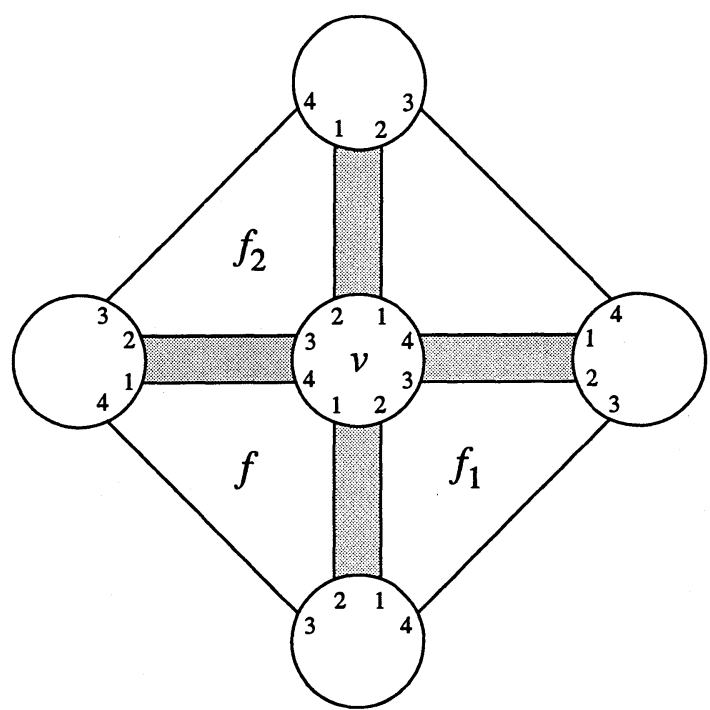

Figure 3.6. 
Let $f_{1}, f_{2}, f$ be the faces indicated in Figure 3.6. Theorem 5.2 guarantees that the 12-edges of $f_{1}$ and $f_{2}$ are not parallel on $G_{T}$ and that the 23-edges of $f_{1}$ and $f_{2}$ are not parallel. An argument similar to the proof of Theorem 5.17 shows that the 34-edges of $f_{1}$ and $f_{2}$ are not parallel on $G_{T}$. The argument of Theorem 6.11 now gives a contradiction.

In all cases we have arrived at a contradiction with our assumption that there is no 41-Scharlemann cycle of length at most 4 . This completes the proof of Theorem 3.4.

Theorem 3.5. In Case $A$ of Corollary $2.4 G_{Q}$ contains a 23-Scharlemann cycle of length at most 4 .

Proof. This follows by applying Theorem 3.4 to the graph $G_{Q}^{\prime}$ obtained from $G_{Q}$ by interchanging labels 1 and 2 and labels 3 and 4 .

Proof of Theorem 3.1. This follows from Corollary 2.4, Theorems 3.3, 3.4 and 3.5, and Theorem 5.10.

\section{The Final Argument.}

By Theorem 3.1, we have the following three possibilities for $\left(m_{1}, m_{2}, m_{3}, m_{4}\right):(2,2,3,3),(2,3,3,3)$ and $(2,3,3,4)$. In this section we will show that each case leads to a contradiction. This completes the proof that our assumption that $t=4$ was impossible.

(2,2,3,3). Here $\Lambda$ contains no $(12,34)$ - or $(23,41)$-bigon (by Theorem 6.9 and Lemma 5.5), and no 34- or 41-Scharlemann cycle of order 2 (by Theorem 5.7). Thus any bigon in $\Lambda$ is a 12- or 23-Scharlemann cycle of order 2 . Hence we cannot have 3 bigons of $\Lambda$ incident at a vertex, contradicting Corollary 7.3.

(2,3,3,3). As in the previous case, $\Lambda$ contains no (12,34)-bigon and no 34-Scharlemann cycle of order 2 . Also, $\Lambda$ contains no $(23,41)$-bigon (by Theorem 5.12), and no 23- or 41-Scharlemann cycle of order 2 (by Theorem 5.7). Thus the only bigons in $\Lambda$ are 12-Scharlemann cycles of order 2 . Again this contradicts Corollary 7.3.

$\underline{(2,3,3,4)}$. Here the only possible bigons in $\Lambda$ are 12-Scharlemann cycles of 
order 2 and $(23,41)$-bigons. (Note that if we had a 41-Scharlemann cycle of order 2 then we would be in the first case above.) Hence, by Corollary 7.3, $\Lambda$ contains a (23,41)-bigon. By Theorem 6.3, the following lemma completes the proof.

Lemma 4.1. $\Lambda$ contains a (23,41)-3-gon.

Proof. By Theorem 5.1, $\Lambda$ cannot contain three parallel bigons. Also, by Corollary 5.4, there cannot be two $(23,41)$-bigons incident to the same vertex at the same label-pair. It follows that if there are three bigons incident at a vertex then they are as illustrated in Figure 4.1, (i), (ii), (iii), (iv) or (v).

By Theorem 7.2, $\Lambda$ contains a special vertex $v$, that is, one of type [5], $[4,2],[4,1,2$,$] or [3,4]$ (see Section 7 ). We discuss each of these in turn.

[3,4]. As noted above, the possibilities for the bigons at $v$ are illustrated in Figure 4.1.

In cases (i) and (ii) at least one (in fact, at least two) of the 3-gons incident to $v$ must be $(23,41)$-3-gons.

In cases (iii) and (iv), note that the face $F_{1}$ cannot be a 3 -gon, as it would either have two 12-corners (contradicting Theorem 5.14), or three 12-corners (contradicting Theorem 5.7). Hence $F_{2}$ is a 3 -gon, and we are done.

Finally, in case (v), at least one of the faces $F_{1}$ and $F_{2}$ is a 3-gon.

[4,1,2]. The four bigons incident at $v$ must be as shown in Figure 4.2.

Let $F_{1}, F_{2}, F_{3}, F_{4}$ be the faces indicated.

If either $F_{2}$ or $F_{4}$ is a 3 -gon then we are done. Hence at least one of $F_{2}, F_{4}$ is a 4-gon. By Theorem 5.14, this 4-gon cannot have three 23-corners, nor two 41-corners. Hence we get a configuration of the form shown in Figure 4.3.

But this is impossible by Theorem 5.21.

[4,2]. Again $v$ is as shown in Figure 4.2.

If one of the two 3-gons incident to $v$ is $F_{2}$ or $F_{4}$ then we are done. So we may suppose that $F_{1}$ and $F_{3}$ are 3 -gons. In particular, $F_{1}$ is a 34 Scharlemann cycle.

Let the two edge classes of 34-edges on $G_{T}$ be I and II (the 12Scharlemann cycle guarantees there are at most 2 classes of 34-edges). By Theorem 5.3, the edges $e_{1}$ and $e_{2}$ are in the same class, say II. If $F_{3}$ were a 34-Scharlemann cycle, then the edges $e_{3}$ and $e_{4}$ would be 34-edges in class I, 
Dehn surgeries on knots creating essential tori, II

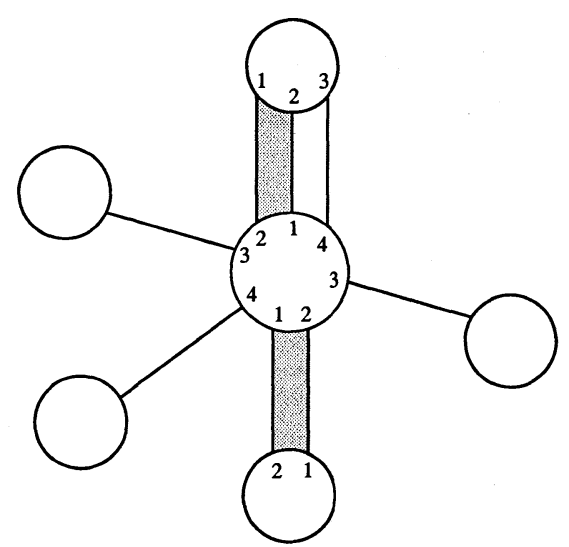

(i)

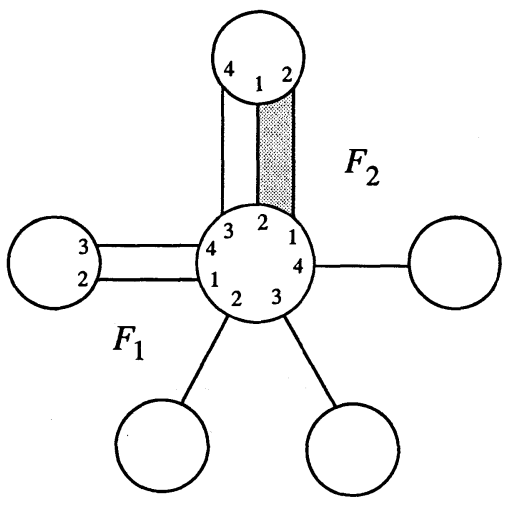

(iii)

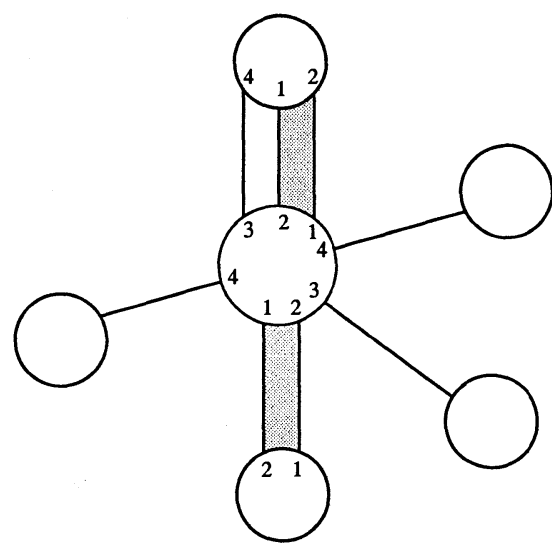

(ii)

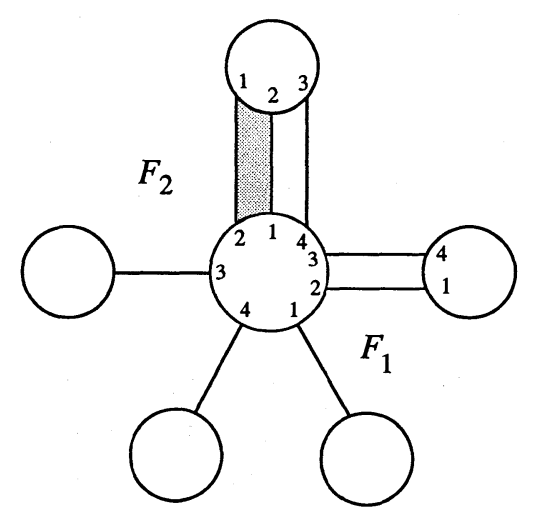

(iv)

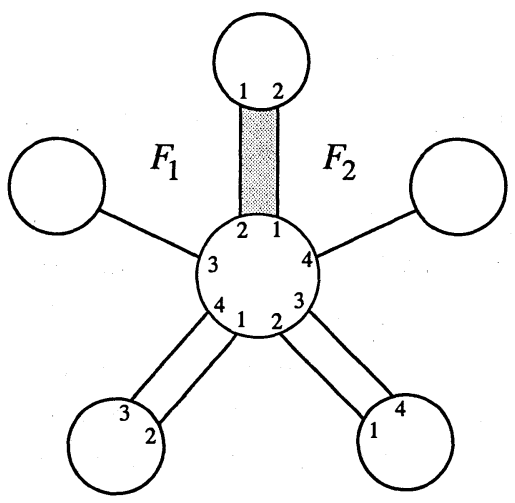

(v)

Figure 4.1. 


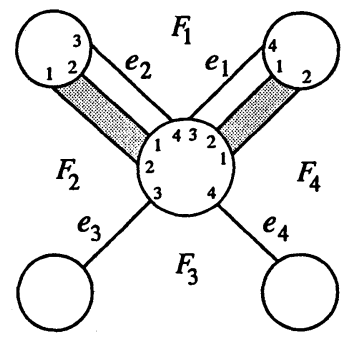

Figure 4.2.

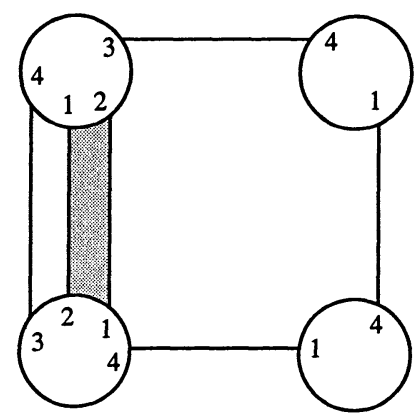

Figure 4.3.

by Theorem 5.2. But then $F_{1}$ and $F_{3}$ would contradict Theorem 5.8 . Hence $F_{3}$ is a $(12,34)$-3-gon, and therefore, by Theorem 5.14 , has two 34-corners and one 12-corner. Hence either $e_{3}$ or $e_{4}$ is a 34-edge, and is in class I by Theorem 5.2. But this contradicts Theorem 5.9.

[5]. Since the only bigons in $\Lambda$ are 12 -Scharlemann cycles and $(23,41)$-bigons this case cannot occur.

\section{Ruling out configurations in $G_{Q}$.}

In this section we use a variety of topological and combinatorial arguments to show that certain configurations in $G_{Q}$ cannot occur.

Recall the following definition from [GLu1]. Let $\sigma$ be a Scharlemann cycle of $G_{Q}$. Suppose that $\sigma$ is immediately surrounded by a cycle $\kappa$ in $G_{Q}$, that is, each edge of $\kappa$ is immediately parallel to an edge of $\sigma$. See Figure 5.1. Then $\kappa$ is called an extended Scharlemann cycle.

Theorem 5.1. $G_{Q}$ does not contain an extended Scharlemann cycle. 


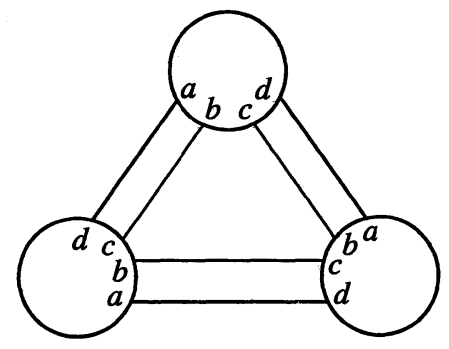

Figure 5.1.

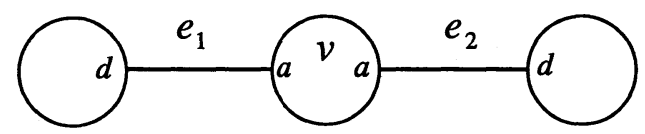

Figure 5.2.

Proof. This is Theorem 3.2 of [GLu1].

Theorem 5.2. Let $e_{1}, e_{2}$ be edges of $G_{Q}$ with the same pair of labels, which are incident to the same vertex $v$ with the same label. See Figure 5.2. Then $e_{1}$ and $e_{2}$ are not parallel on $G_{T}$.

Proof. If $e_{1}$ and $e_{2}$ are parallel on $G_{T}$ then they cobound $q+1$ parallel edges on $G_{T}$, where $q$ is the number of boundary components of $Q$. The argument of [GLi,p.130,Case (2)] now constructs a cable space in $E(K)$, contradicting our assumptions on $K$.

We introduce some notation which will be used in the remainder of this section and in Section 6.

Note that a fat vertex of $G_{T}$ is a component of $\widehat{T} \cap V_{\gamma}$. Then $H_{a b}$ will denote the 1-handle consisting of that part of $V_{\gamma}$ between consecutive components $a$ and $b$ of $\widehat{T} \cap V_{\gamma}$.

$X$ and $X^{\prime}$ will denote the closures of the components of $E(K)-T$. In particular, $\partial X$ and $\partial X^{\prime}$ are surfaces of genus 3. Note that $X$ and $X^{\prime}$ are irreducible and (by our hypothesis on $K$ ) atoroidal. Similarly, $\widehat{X}$ and $\widehat{X}^{\prime}$ will denote the closures of the corresponding components of $K(\gamma)-\widehat{T}$.

Finally, nhd(...) or $N(\ldots)$ will denote a regular neighborhood of $(\ldots)$, with $\operatorname{nhd}_{S}(\ldots)$ indicating that the regular neighborhood is to be taken in $S$. 


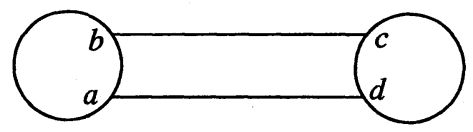

Figure 5.3.

Theorem 5.3. Suppose that $G_{Q}$ contains a bc-Scharlemann cycle. Then in any two occurrences in $\Lambda$ of an (ab,cd)-bigon (Figure 5.3), the corresponding edges are parallel on $G_{T}$.

Proof. Assume for contradiction that there are faces $f_{1}$ and $f_{2}$ in $\Lambda$ as in Figure 5.3 for which either the $b c$-edges are not parallel or the $d a$-edges are not parallel on $G_{T}$. Then in fact neither the $b c$-edges nor the $d a$-edges can be parallel on $G_{T}$. For, suppose the $b c$-edges (say) are parallel on $G_{T}$, while the $d a$-edges are not. Then, banding the disks $f_{1}$ and $f_{2}$ together along the co-core of the parallelism on $T$ between the $b c$-edges, we get a disk whose boundary can be isotoped off the 1-handles $H_{a b}$ and $H_{c d}$ to form an essential curve on $T$. This contradicts the incompressibility of $T$.

Let $A$ be the annulus obtained by taking $f_{1} \cup f_{2} \cup H_{a b} \cup H_{c d}$ and radially shrinking $H_{a b}, H_{c d}$ to their cores. Since $K(\gamma)$ contains no embedded Klein bottle (this follows for homological reasons from the fact that $\Delta(\gamma, \mu)=2$, see Lemma 6.2 of [GLu1]), we see that surgering $\widehat{T}$ along $A$ produces two tori which miss $K_{\gamma}$. The minimality of $\widehat{T}$ then implies that these two tori bound disjoint solid tori $V_{1}$ and $V_{2}$ in $\widehat{X}$. Thus $\widehat{X}=V_{1} \cup_{A} V_{2}$ is a Seifert fiber space over the disk with two exceptional fibers. Furthermore, the Seifert fiber is isotopic to a component of $\partial A$.

Let $\sigma$ be a $b c$-Scharlemann cycle and $f_{3}$ the face of $G_{Q}$ that it bounds. Since $A$ contains non-parallel $d a$-edges, the edges of $\sigma$ lie in an annulus $C \subset$ $\widehat{T}$ where $\partial C$ is isotopic to $\partial A$ and which may be taken to be disjoint from the $d a$-edges of $G_{T}$. Let $V_{3}=\operatorname{nhd}\left(C \cup H_{b c} \cup f_{3}\right)$. Then $\left|\partial V_{3} \cap K_{\gamma}\right|=2$, and hence $V_{3}$ is a solid torus, by our minimality assumption on $\widehat{T}$. Let $\partial V_{3}=C \cup C^{\prime}$. Then again $\widehat{T}^{\prime}=(\widehat{T}-C) \cup C^{\prime}$ is a torus with $\left|\widehat{T}^{\prime} \cap K_{\gamma}\right|=2$. Hence $\widehat{X}^{\prime}$ may be written as $V_{3} \cup_{C^{\prime}} V_{4}$, where $V_{4}$ is also a solid torus. Therefore $\widehat{X}^{\prime}$ is also a Seifert fiber space over the disk with two exceptional fibers, and the Seifert fiber is isotopic to a component of $\partial C$. Since $\partial C$ and $\partial A$ are isotopic in $\widehat{T}$, $K(\gamma)$ is a Seifert fiber space over the 2-sphere with four exceptional fibers.

By an isotopy of $\partial A$ we may assume that $\partial V_{1}$ contains $C$. Let $M=$ $V_{1} \cup_{C} V_{3}$. Then $\partial M$ is essential in $K(\gamma)$ and $\left|\partial M \cap K_{\gamma}\right|=2$, contradicting the minimality of $\widehat{T}$. 
Corollary 5.4. Suppose that $G_{Q}$ contains a bc-Scharlemann cycle. Then $\Lambda$ cannot contain two $(a b, c d)$-bigons whose ab-corners occur at the same vertex.

Proof. This follows from Theorems 5.3 and 5.2.

Lemma 5.5. The edges of a Scharlemann cycle in $G_{Q}$ do not lie in a disk in $\widehat{T}$.

Proof. This is Lemma 3.1 of [GLu1].

Lemma 5.6. The edges of a Scharlemann cycle in $G_{Q}$ of length 2 or 3 lie in an annulus in $\widehat{T}$.

Proof. For a Scharlemann cycle of length 2 this is clear. For length 3 it is proved in Lemma 3.7 of [GLu1].

Theorem 5.7. Suppose that $G_{Q}$ contains an ab-Scharlemann cycle. Then $G_{Q}$ does not contain cd-Scharlemann cycles of distinct lengths.

Proof. Let $\sigma_{1}$ and $\sigma_{2}$ be $c d$-Scharlemann cycles of distinct lengths. By Lemma 5.5, the existence of the $a b$-Scharlemann cycle forces the edges of $\sigma_{1}$ and $\sigma_{2}$ to lie in a single essential annulus $A \subset \widehat{T}$. Consider the torus obtained by attaching disks to $\partial A$ and surgering the resulting 2-sphere using the 1-handle $H_{c d}$. Then the boundaries of the faces of $G_{Q}$ bounded by $\sigma_{1}$ and $\sigma_{2}$ would be disjoint, homologically distinct simple closed curves on this torus, a contradiction.

Theorem 5.8. Suppose that $G_{Q}$ contains an ab-Scharlemann cycle, and a cd-Scharlemann cycle of length 3 . Then there are edge classes I and II in $G_{T}$ such that any cd-Scharlemann cycle has exactly one edge in class I and two edges in class II.

Proof. As in the proof of Theorem 5.7, there is an essential annulus $A \subset$ $\widehat{T}$ such that the edges of any $c d$-Scharlemann cycle lie in $A$. Thus there are edges classes I and II such that any edge of any $c d$-Scharlemann cycle 


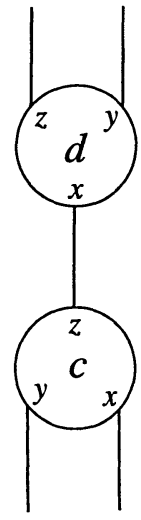

Figure 5.4.

is in class I or II. Suppose without loss of generality that the given $c d$ Scharlemann cycle of length $3, \sigma_{1}$ say, has one edge in class I and two edges in class II. Let $\sigma_{2}$ be another $c d$-Scharlemann cycle, necessarily of length 3 by Theorem 5.7. Suppose for contradiction that $\sigma_{2}$ has two edges in class I and one edge in class II. Let $f_{1}, f_{2}$ be the faces of $G_{Q}$ bounded by $\sigma_{1}, \sigma_{2}$ respectively. Let $N=\operatorname{nhd}\left(A \cup H_{c d} \cup f_{1} \cup f_{2}\right)$. An easy computation shows that $H_{1}(N) \cong \mathbb{Z}_{3}$. Since $K(\gamma)-N$ contains (an isotopic copy of) the incompressible torus $\widehat{T}$, it follows that $\partial N$ is a 2-sphere that does not bound a 3-ball in $K(\gamma)$. But this contradicts [GLu2].

We will use the following notation in the proof of Theorem 5.9 below, and also in the proofs of Theorems 5.21 and 6.14. Let $a$ be a vertex of $G_{T}$ and let $\alpha, \beta$ be labels at $a$. Then $s(a ; \alpha, \beta)$ will denote the arc in the boundary of vertex $a$ that runs clockwise from $\alpha$ to $\beta$.

Theorem 5.9. Suppose that $G_{Q}$ contains an ab-Scharlemann cycle, and a cd-Scharlemann cycle of length 3. Let I and II be the edge classes in Theorem 5.8. Then any $(a b, c d)$-face of $G_{Q}$ containing a cd-edge contains a cd-edge in class II.

Proof. We label the vertices of the $c d$-Scharlemann cycle $x, y, z$ so that in $G_{T}$ they appear as in Figure 5.4.

Let $f$ be an $(a b, c d)$-face of $G_{Q}$ containing a $c d$-edge $e$, which we may suppose is in class I. Let $e_{1}, e_{2}$ be the edges of $f$ adjacent to $e$. See Figure 5.5.

Let $u_{c}$ denote the label $u$ at vertex $c$ in $G_{T}$ corresponding to the appropriate endpoint of $e$, etc. Since $e$ is in class I, either $u_{c} \in s(c ; y, z)$ or 


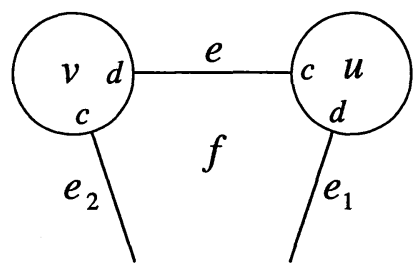

Figure 5.5.

$v_{d} \in s(d ; y, x)$. Thus either $u_{d} \in s(d ; z, y)$ or $v_{c} \in s(c ; x, y)$, implying that either $e_{1}$ or $e_{2}$ is a $c d$-edge in class II.

Theorem 5.10. $G_{Q}$ does not contain Scharlemann cycles of even order on disjoint label-pairs.

Proof. Suppose that $G_{Q}$ contains $a b$ - and $c d$-Scharlemann cycles of orders $2 p$, $2 q$ respectively. Let $f_{1}, f_{2}$ be the faces bounded by these Scharlemann cycles. Suppose that $f_{1}$ and $f_{2}$ lie in $X$. Let $W=\operatorname{nhd}_{\widehat{X}}\left(\widehat{T} \cup H_{a b} \cup H_{c d} \cup f_{1} \cup f_{2}\right)$. Then $\partial W=\widehat{T} \cup T_{1}$, where $T_{1}$ is a torus. By our hypothesis on $E(K)$, $T_{1}$ bounds a solid torus $V$ in $X$; let $D$ be a meridian disk of $V$. Then $\widehat{X}=W \cup N(D) \cup 3$-cell.

Since $\Delta=2, H_{1}\left(K(\gamma) ; \mathbb{Z}_{2}\right)=0$. Hence

$$
0=H_{1}\left(K(\gamma), \widehat{X}^{\prime} ; \mathbb{Z}_{2}\right) \cong H_{1}\left(\widehat{X}, \widehat{T} ; \mathbb{Z}_{2}\right) \text { (by excision). }
$$

But $H_{1}\left(\widehat{X}, \widehat{T} ; \mathbb{Z}_{2}\right)$ is generated by the elements $\alpha_{1}, \alpha_{2}$ represented by the cores of the 1-handles $H_{a b}$ and $H_{c d}$, with relations given by $\partial f_{1}, \partial f_{2}$ and $\partial D$. Since $\left[\partial f_{1}\right]=2 p \alpha_{1}$ and $\left[\partial f_{2}\right]=2 q \alpha_{2}$ are zero $\bmod 2$, we have that $H_{1}\left(\widehat{X}, \widehat{T} ; \mathbb{Z}_{2}\right) \cong \mathbb{Z}_{2} \oplus \mathbb{Z}_{2} /([\partial D])$ has dimension $\geq 1$, a contradiction.

Let $f_{1}, f_{2}, f_{3}$ be faces of $G_{Q}$ that lie on the same side of $T$, so are contained in (say) $X$. We shall say that $f_{1}, f_{2}, f_{3}$ are independent if $\partial f_{1}, \partial f_{2}, \partial f_{3}$ are homologically independent curves on $\partial X$.

Lemma 5.11. Let $f_{1}, f_{2}$ be faces of $G_{Q}$ bounded by Scharlemann cycles of lengths $p$ and $q$ on label-pairs ab, cd respectively. Let $f$ be an $(a b, c d)$-face of $G_{Q}$ with $m$ ab-corners and $n$ cd-corners $(m, n \neq 0)$. Then $f_{1}, f_{2}, f$ are independent unless $m=p, n=q$.

Proof. Cutting $\partial X$ along $\partial f_{1}$ and $\partial f_{2}$ gives a 4-punctured torus $T_{0}$. If $f_{1}, f_{2}, f$ are not independent then $\partial f$ bounds a disk $D$ on the corresponding 

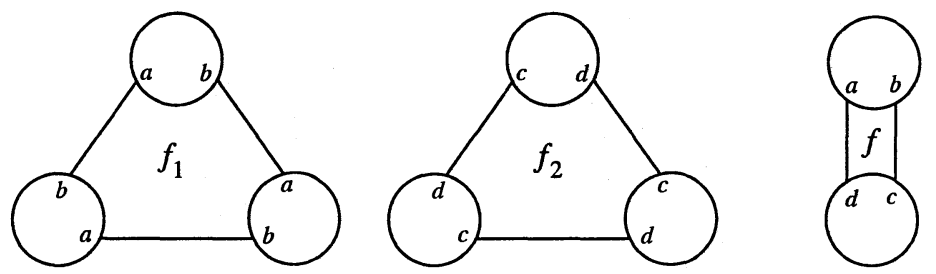

Figure 5.6.

(unpunctured) torus $\widehat{T}_{0}$. Then $D \cap T_{0}$ gives a relation in $H_{1}(\partial X)$ of the form

$$
[\partial f]=\varepsilon_{1}\left[\partial f_{1}\right]+\varepsilon_{2}\left[\partial f_{2}\right]
$$

where $\varepsilon_{i} \in\{-1,0,1\}, i=1,2$.

The composition

$$
\partial X \rightarrow \widehat{T} \cup H_{a b} \cup H_{c d} \rightarrow\left(\widehat{T} \cup H_{a b} \cup H_{c d}\right) / \widehat{T},
$$

where the first map is inclusion and the second is the quotient map, induces on homology an epimorphism $H_{1}(\partial X) \rightarrow \mathbb{Z} \oplus \mathbb{Z}$, under which

$$
\begin{aligned}
{\left[\partial f_{1}\right] } & \longmapsto(p, 0) \\
{\left[\partial f_{2}\right] } & \longmapsto(0, q) \\
\text { and }[\partial f] & \longmapsto(m, n) .
\end{aligned}
$$

For the latter, recall that an $(a b, c d)$-face of $G_{Q}$ by definition abuts vertices of $G_{Q}$ all of which have the same sign. Then (5.1) implies $m=\varepsilon_{1} p, n=\varepsilon_{2} q$. Since $m, n \neq 0$, we must have $m=p, n=q$.

Theorem 5.12. $G_{Q}$ does not contain an ab-Scharlemann cycle of length 3 , a cd-Scharlemann cycle of length 3 , and an $(a b, c d)$-bigon.

Proof. Suppose, for contradiction, that $G_{Q}$ contains faces $f_{1}, f_{2}, f$ as shown in Figure 5.6. By Lemma 5.11, $f_{1}, f_{2}, f$ are independent. Hence, if $\widehat{X}$ is the complementary component of $\widehat{T}$ in $K(\gamma)$ containing $H_{a b}$ and $H_{c d}$, we have $\widehat{X}=\operatorname{nhd}_{\widehat{X}}\left(\widehat{T} \cup H_{a b} \cup H_{c d} \cup f_{1} \cup f_{2} \cup f\right) \cup 3$-cell.

Now $H_{1}(\widehat{X}, \widehat{T})$ is generated by $\alpha_{1}, \alpha_{2}$, the elements represented by the cores of the 1-handles $H_{a b}, H_{c d}$, and $\partial f_{1}, \partial f_{2}, \partial f$ give the relations $3 \alpha_{1}=0$, $3 \alpha_{2}=0, \alpha_{1}+\alpha_{2}=0$. Hence $H_{1}(\widehat{X}, \widehat{T}) \cong \mathbb{Z}_{3}$.

Let $\kappa=\left[K_{\gamma}\right] \in H_{1}(K(\gamma))$. Let $\delta$ be a simple closed curve on $\partial E(K)$ such that $\Delta(\gamma, \delta)=1$. Then $\delta$ represents $\kappa \in H_{1}(K(\gamma))$. Hence

$$
H_{1}(K(\gamma)) /(\kappa) \cong H_{1}(E(K)) /([\gamma],[\delta])=0 .
$$


But the map

$$
H_{1}(K(\gamma)) \rightarrow H_{1}\left(K(\gamma), \widehat{X}^{\prime}\right) \cong H_{1}(\widehat{X}, \widehat{T}) \quad(\text { excision })
$$

takes $\kappa$ to $\alpha_{1}+\alpha_{2}$. Since $H_{1}(\widehat{X}, \widehat{T}) /\left(\alpha_{1}+\alpha_{2}\right) \cong \mathbb{Z}_{3}$, this is a contradiction.

Lemma 5.13. Let $f_{1}, f_{2}, f$ be independent $(a b, c d)$-faces of $G_{Q}$, where $f$ is the face bounded by an ab-Scharlemann cycle. Suppose that $f_{i}$ has $n_{i} c d-$ corners, $i=1,2$. Then $\left(n_{1}, n_{2}\right)=1$.

Proof. Since $f_{1}, f_{2}, f$ are independent, $\widehat{X}=\operatorname{nhd}_{\widehat{X}}\left(\widehat{T} \cup H_{a b} \cup H_{c d} \cup f_{1} \cup f_{2} \cup\right.$ $f) \cup B^{3}$. Let $W=\operatorname{nhd}_{\widehat{X}}\left(\widehat{T} \cup H_{a b} \cup f\right)$. Then $\partial W=\widehat{T} \cup \widehat{T}_{1}$, where $\widehat{T}_{1}$ is a torus such that $\left|\widehat{T}_{1} \cap K_{\gamma}\right|=2$. By our minimality assumption on $\widehat{T}$, and the irreducibility of $K(\gamma)$ [GLu2], $\widehat{T}_{1}$ bounds a solid torus $V$ in $\widehat{X}$.

Now consider the handle decomposition of $\widehat{X}$ dual to the one described above; in particular, let $D_{c d}$ be the co-core of the 1-handle $H_{c d}$, and let $H_{f_{1}}, H_{f_{2}}$ be the 1-handles dual to the 2-handles $N\left(f_{1}\right), N\left(f_{2}\right)$. Then

$$
V=B^{3} \cup H_{f_{1}} \cup H_{f_{2}} \cup N\left(D_{c d}\right) .
$$

Since $\partial D_{c d} \cdot \partial f_{i}=n_{i}, i=1,2$, we get $H_{1}(V) \cong \mathbb{Z} \oplus \mathbb{Z}_{\left(n_{1}, n_{2}\right)}$. Hence $\left(n_{1}, n_{2}\right)=$ 1.

Theorem 5.14. Assume that $G_{Q}$ contains Scharlemann cycles of lengths $p$ and $q$ on label-pairs ab, cd respectively. Let $f$ be an $(a b, c d)$-face of $G_{Q}$ with $m$ ab-corners and $n$ cd-corners $(m, n \neq 0)$. Then either $(m, p)=1=(n, q)$, or $m=p, n=q$.

Proof. Suppose the ordered pairs $(m, n)$ and $(p, q)$ are unequal. Then by Lemma $5.11 f_{1}, f_{2}, f$ are independent. Applying Lemma 5.13 twice gives $(m, p)=1=(n, q)$.

The following lemma will be used in the proof of Theorem 5.16 below. It will also be used in the proofs of Lemma 5.19 and Theorem 5.20.

Lemma 5.15. Let $W$ be a compact 3-dimensional submanifold of $K(\gamma)$. Then $H_{1}(W)$ contains no 2-torsion. 


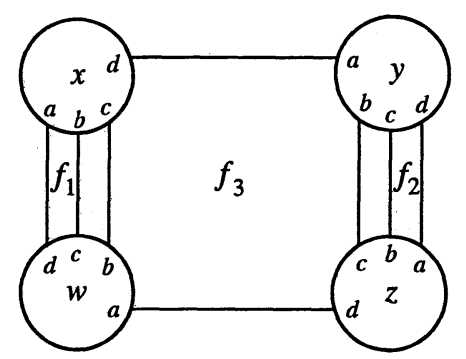

Figure 5.7.

Proof. Assume that $W$ is connected. Let $W^{\prime}=\overline{K(\gamma)-W}$. Since $\Delta(\gamma, \mu)=$ $2, H_{1}(K(\gamma)) \cong \mathbb{Z}_{m}, m$ odd. The Mayer-Vietoris theorem gives an exact sequence

$$
0 \rightarrow H_{1}(\partial W) \rightarrow H_{1}(W) \oplus H_{1}\left(W^{\prime}\right) \rightarrow H_{1}(K(\gamma)) \rightarrow 0 .
$$

Since $H_{1}(\partial W)$ is free abelian, the torsion subgroup of $H_{1}(W)$ maps injectively into $H_{1}(K(\gamma))$. The result follows.

Theorem 5.16. $G_{Q}$ does not contain a configuration as in Figure 5.7.

Proof. Let $f_{1}, f_{2}, f_{3}$ be the faces indicated.

By Theorem 5.3, the existence of the $b c$-Scharlemann cycle implies that the corresponding edges of $f_{1}$ and $f_{2}$ are parallel on $G_{T}$. In particular, the $b c$-edges of $f_{1}$ and $f_{2}$ are parallel on $G_{T}$. It follows that the $b c$-edges of $f_{3}$ are also parallel on $G_{T}$, since otherwise, as in the first paragraph of the proof of Theorem 5.3, the disks bounded by the two $b c$-Scharlemann cycles in Figure 5.7 could be banded together and isotoped off $H_{b c}$ to give a compressing disk for $T$.

We claim that the $d a$-edges of $f_{3}$ are not parallel on $G_{T}$. For otherwise, the edges of $f_{3}$ would lie in a disk $D \subset \widehat{T}$ (containing the fat vertices of $G_{T}$ ). Let $W=\operatorname{nhd}\left(D \cup H_{b c} \cup H_{d a} \cup f_{3}\right)$. Then $H_{1}(W) \cong \mathbb{Z} \oplus \mathbb{Z}_{2}$, contradicting Lemma 5.15 .

Without loss of generality the $b c$-edges of $f_{1}$ and $f_{3}$ appear on $G_{T}$ as in Figure 5.8.

The ordering of the labels $w, x, y$ around vertex $b$ determines the (reverse) ordering of these labels around vertex $a$. Then the $d a$-edges of $f_{1}$ and $f_{3}$ determine the ordering of the labels $w, x, z$ around vertex $d$ as shown in Figure 5.8. But this is inconsistent with the ordering of these labels around vertex $c$. 

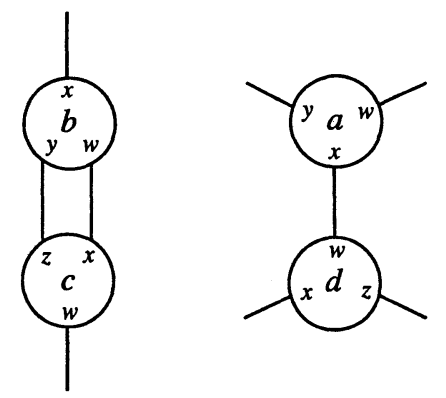

Figure 5.8.

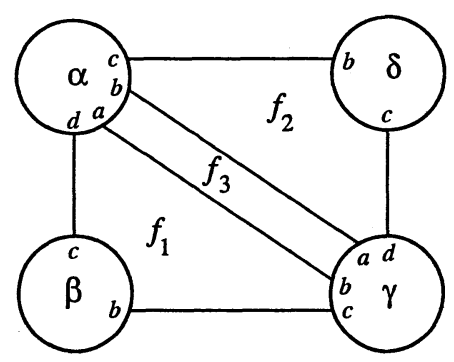

Figure 5.9.

Theorem 5.17. Suppose that $G_{Q}$ contains a configuration as in Figure 5.9. Then no two corresponding edges (e.g., the ab-edges) are parallel on $G_{T}$.

Proof. The $a b$-edges cannot be parallel on $G_{T}$ by Lemma 5.5 .

Assume for contradiction that the $b c$-edges are parallel on $G_{T}$. Up to homeomorphism (and changing of labels) of $G_{T}$ the edges of the configuration appear as in Figure 5.10(i) (where the $c d$-edges may or may not be parallel).

Let $f_{1}, f_{2}, f_{3}$ be the faces indicated in Figure 5.9. Let $f$ be the disk obtained by band summing $f_{1}$ and $f_{2}$ along the co-core of the parallelism between the $b c$-edges (see Figure 5.10(ii)). Slide $f$ over and off $H_{b c}$ as in Figure 5.10(iii). We see that $\partial f$ now divides $\partial H_{d a}-\widehat{T}$ into two disks. If $C$ is one of these disks then $A=f \cup C$ is an annulus. One component of $\partial A$ is formed by the $a b$-edges of the configuration and hence is essential on $\widehat{T}$. Since $\widehat{T}$ is essential the other component of $\partial A$ must be essential on $\widehat{T}$. Thus $\widehat{T}=B_{1} \cup_{\partial A} B_{2}$ where $B_{1}$ and $B_{2}$ are annuli. Furthermore, by picking $C$ correctly we have that $\left|B_{1} \cap K_{\gamma}\right|=\left|B_{2} \cap K_{\gamma}\right|=2$ (there are no Klein bottles in $K(\gamma)$ by Lemma 6.2 of [GLu1]). Let $\widehat{T}_{1}=A \cup B_{1}, \widehat{T}_{2}=A \cup B_{2}$. Since 


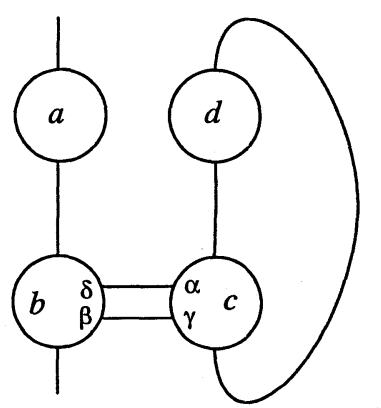

(i)

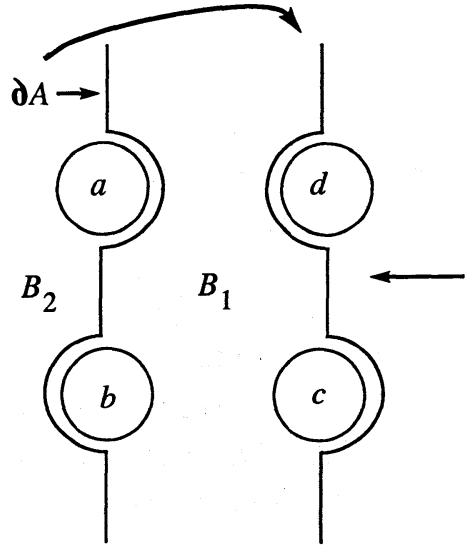

(iv)

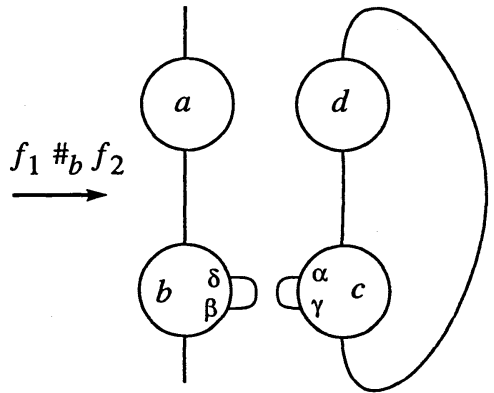

(ii)

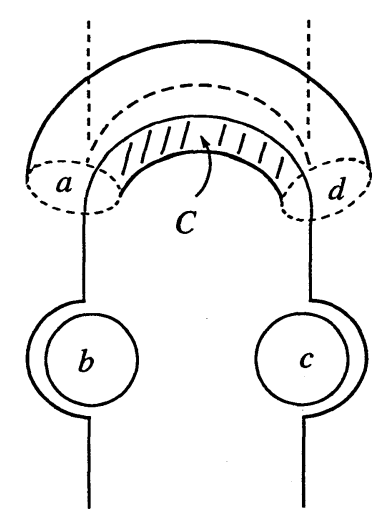

(iii)

Figure 5.10. 
$T_{1}$ and $T_{2}$ each intersect $K_{\gamma}$ fewer times than $\widehat{T}$, they must bound solid tori $V_{1}$ and $V_{2}$. Thus the side of $\widehat{T}$ containing $A$ can be written $V_{1} \cup_{A} V_{2}$ and must be Seifert fibered over the disk with two exceptional fibers. Expand $V_{1}$ to include $H_{d a}$. Let $N=\operatorname{nhd}\left(V_{1} \cup H_{a b} \cup f_{3}\right)$. By Lemma 3.7 of [GLu1] and the above, we see that $N$ is a Seifert fiber space over the disk with two exceptional fibers. In particular, $\partial N$ is essential in $K(\gamma)(\partial N$ cannot compress in $K(\gamma)-N$; to see this consider the intersection of a compressing disk with $\left.B_{2}\right)$. But $\left|\partial N \cap K_{\gamma}\right|<\left|\widehat{T} \cap K_{\gamma}\right|$, contradicting the minimality of $\widehat{T}$.

The argument for the case when the $c d$-edges are parallel is similar and is pictured in Figure 5.11.

Theorem 5.18. Suppose that $G_{Q}$ contains a da-Scharlemann cycle of length 2 or 3 . Then $G_{Q}$ does not contain a configuration as in Figure 5.9.

Proof. Suppose that $G_{Q}$ contains a configuration as in Figure 5.9.

By Theorem 5.17, corresponding edges are not parallel on $G_{T}$. This implies that up to homeomorphism of $G_{T}$, and possibly interchanging the labels $\beta$ and $\delta$, the edges appear on $G_{T}$ as in Figure 5.12(i) or (ii). Note that once the labelling around vertex $b$ of $G_{T}$ is chosen, the arcs of $\left(\partial f_{1} \cup \partial f_{2}\right) \cap H_{b c}$ determine the labelling on vertex $c$.

Assume for contradiction that $G_{Q}$ also contains a $d a$-Scharlemann cycle $\sigma$ of length $p$, where $p=2$ or 3 . Let $f$ be the face of $G_{Q}$ bounded by $\sigma$.

We claim that $f_{1}, f_{2}, f$ are independent. To see this, let $\alpha_{1}, \alpha_{2}$ be the simple closed curves on $\partial X$ that are the boundaries of the co-cores of the 1-handles $H_{d a}, H_{b c}$, respectively. Let $\alpha_{3}$ be the simple closed curve on $T$ indicated in Figure 5.12. Then one easily computes that the intersection numbers of $\partial f_{1}$ etc. with $\alpha_{1}, \alpha_{2}$ and $\alpha_{3}$ are as follows:

$$
\begin{array}{ll}
\text { case (i) } & \partial f_{1}:(1,2,1) \\
& \partial f_{2}:(1,2,-1) \\
& \partial f:(p, 0,0) \\
& \\
\text { case (ii) } & \partial f_{1}:(1,2,2) \\
& \partial f_{2}:(1,2,-1) \\
& \partial f:(p, 0, q) \text { for some } q .
\end{array}
$$

In both cases the corresponding determinants are non-zero, proving the claim. 


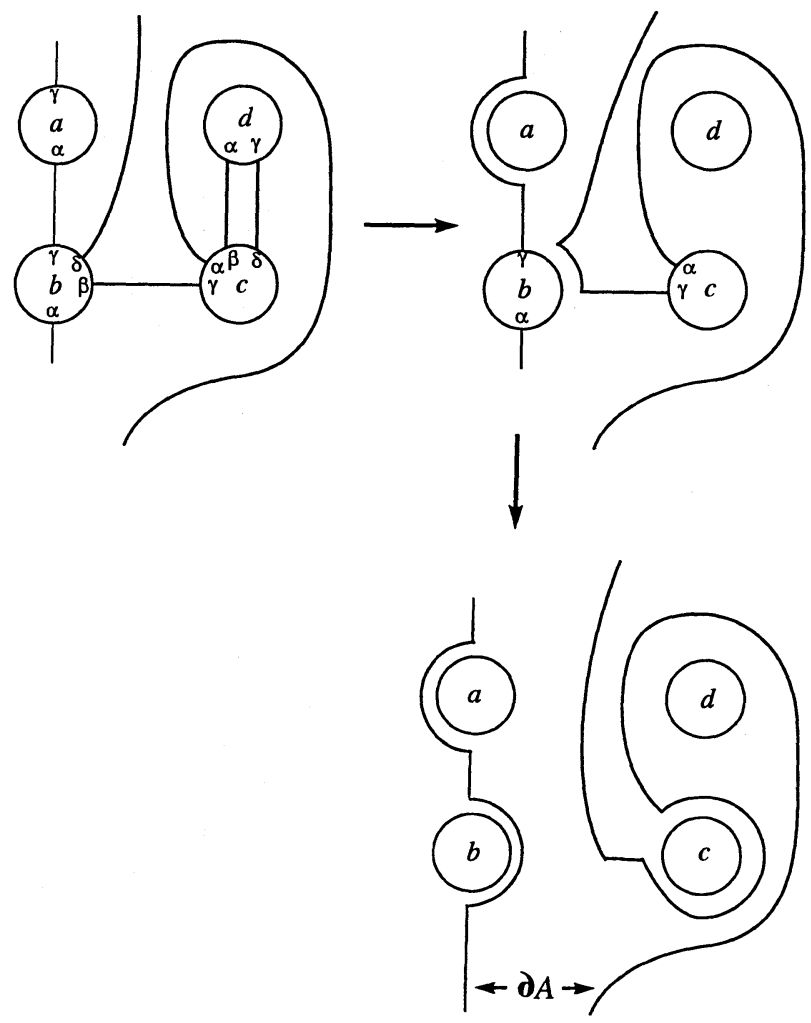

Figure 5.11.

Since each of $f_{1}, f_{2}$ has two $b c$-corners, this contradicts Lemma 5.13 .

Lemma 5.19. Suppose that $G_{Q}$ contains a configuration as in Figure $5.13(i)$ or (ii). Then the cd-edges are parallel on $G_{Q}$.

Proof. Suppose that $G_{Q}$ contains a configuration $\Omega$ as in Figure 5.13(i). (The case of Figure 5.13(ii) is the same. Note that 5.13(ii) becomes (i) by interchanging labels $a, b$ and $c, d$ and applying an orientation-reversing homeomorphism to $\widehat{Q}$.)

Assume for contradiction that the $c d$-eges of $\Omega$ are not parallel on $G_{T}$. Then (by considering the arcs of $\left.\left(\partial f_{1} \cup \partial f_{3}\right) \cap H_{b c}\right)$ one checks that up to homeomorphism the edges of $\Omega$ appear on $G_{T}$ as shown in Figure 5.14.

Let $A \subset \widehat{T}$ be an annulus containing the edges of $\Omega$. Note that $H_{1}(A \cup$ $H_{d a} \cup H_{b c}$ ) has basis $x, y, z$, represented by the cores of $A, H_{d a}$ and $H_{b c}$ 


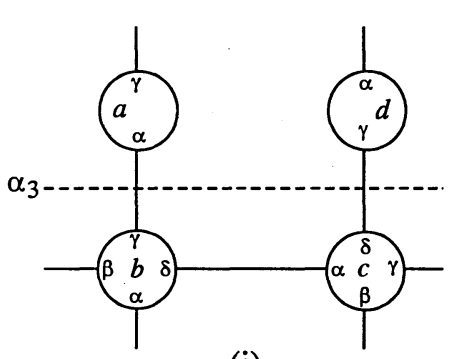

(i)

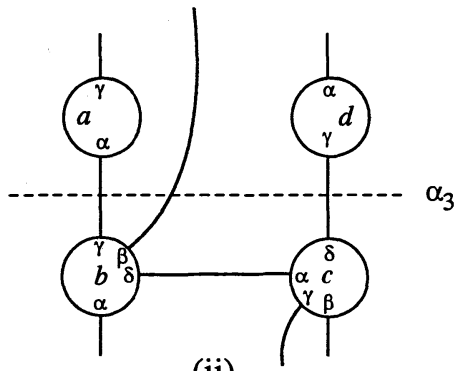

(ii)

Figure 5.12.

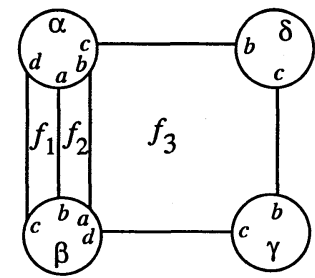

(i)

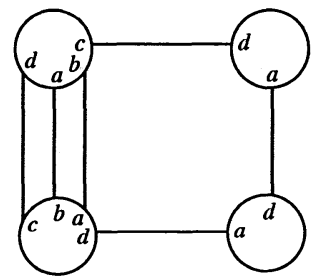

(ii)

Figure 5.13.

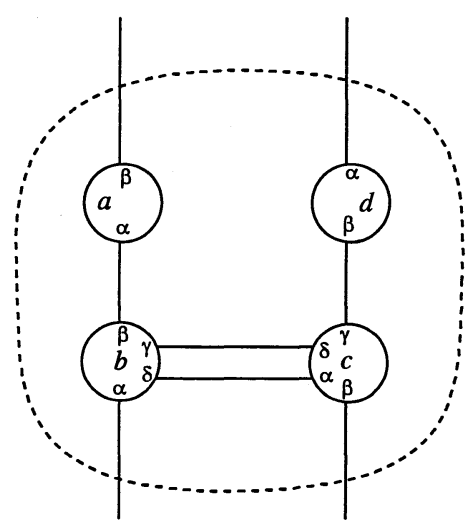

Figure 5.14. 


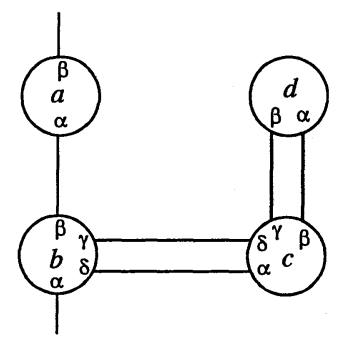

Figure 5.15.

respectively (where the cores of the 1-handles are completed to 1-cycles within the disk indicated in Figure 5.14). Let $N=\operatorname{nhd}\left(A \cup H_{d a} \cup H_{b c} \cup\right.$ $\left.f_{1} \cup f_{3}\right)$. Since $\left[\partial f_{1}\right]=-x+y+z$, and $\left[\partial f_{3}\right]=x+y+3 z$, we see that $H_{1}(N) \cong \mathbb{Z} \oplus \mathbb{Z}_{2}$. But this contradicts Lemma 5.15.

Theorem 5.20. Suppose that $G_{Q}$ contains a configuration as in Figure $5.13(i)$ (resp. (ii)). Then the bc-edges (resp. da-edges) are not parallel on $G_{T}$.

Proof. Suppose that $G_{Q}$ contains a configuration $\Omega$ as in Figure 5.13(i). (Figure 5.13(ii) becomes 5.13(i) after relabelling.)

Assume for contradiction that the $b c$-edges of $\Omega$ are parallel on $G_{T}$. Then, by Lemma 5.19 and considering the arcs of $\left(\partial f_{1} \cup \partial f_{3}\right) \cap H_{b c}$, the edges of $\Omega$ appear on $G_{T}$ as in Figure 5.15.

Let $A \subset \widehat{T}$ be an annulus containing the edges of $\Omega$. Then $H_{1}(A \cup$ $\left.H_{d a} \cup H_{b c} \cup H_{a b}\right)$ has basis $x, y, z, w$ represented by the cores of $A, H_{d a}$, $H_{b c}$ and $H_{a b}$ respectively (and with the same convention as in the proof of Lemma 5.19 above). Let $N=\operatorname{nhd}\left(A \cup H_{c a} \cup H_{b c} \cup H_{a b} \cup f_{1} \cup f_{3} \cup f_{2}\right)$. Since $\left[\partial f_{1}\right]=y+z,\left[\partial f_{3}\right]=x+y+3 z$, and $\left[\partial f_{2}\right]=-x+2 w$, we compute that $H_{1}(N) \cong \mathbb{Z} \oplus \mathbb{Z}_{2}$, contradicting Lemma 5.15.

Theorem 5.21. Suppose that $G_{Q}$ contains a cd-Scharlemann cycle. Then $G_{Q}$ does not contain a configuration as in Figure 5.13(i) or (ii).

Proof. We show that Figure 5.13(i) is impossible; the proof for Figure 5.13(ii) is similar.

So suppose for contradiction that $G_{Q}$ contains a configuration $\Omega$ as in Figure 5.13(i), as well as a $c d$-Scharlemann cycle $\sigma$. 


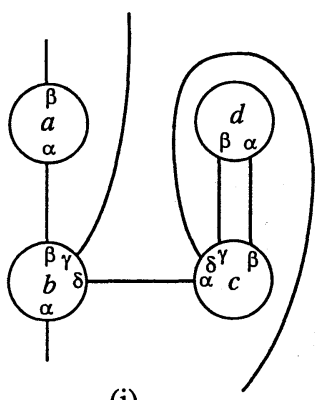

(i)

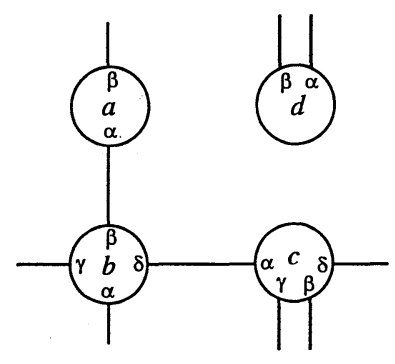

(ii)

Figure 5.16.
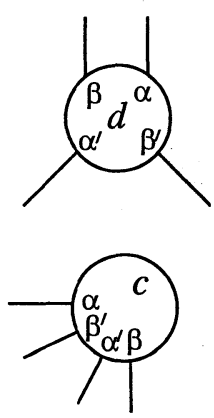

Figure 5.17.

By considering the arcs of $\left(\partial f_{1} \cup \partial f_{3}\right) \cap H_{b c}$ we see that, up to homeomorphism, the edges of $\Omega$ must appear on $G_{T}$ as in Figure 5.16(i) or (ii).

Since the edges of $\sigma$ do not lie in a disk by Lemma 5.5, Figure 5.16(i) is impossible.

So assume the picture is as in Figure 5.16(ii). At vertex $d$ there are edges incident with labels $\alpha$ and $\beta$ that are not pictured in the figure. We denote these incidences by $\alpha^{\prime}$ and $\beta^{\prime}$. Because of the sequential ordering of labels around a vertex and the fact that each label appears exactly twice, $\alpha^{\prime}$ and $\beta^{\prime}$ are either both in $s(d ; \alpha, \beta)$ or both in $s(d ; \beta, \alpha)$. But $\alpha^{\prime} \in s(d ; \beta, \alpha)$ would violate Theorem 5.2. Thus $\alpha^{\prime}, \beta^{\prime} \in s(d ; \alpha, \beta)$. See Figure 5.17.

Similarly, we denote by $\alpha^{\prime}$ and $\beta^{\prime}$ the occurrences of the labels $\alpha$ and $\beta$ at vertex $c$ that are not shown in Figure 5.16(ii). The arcs of $\partial Q \cap H_{c d}$ then dictate that $\alpha^{\prime}, \beta^{\prime} \in s(c ; \beta, \alpha)$, as in Figure 5.17.

Recall that $\sigma$ is a $c d$-Scharlemann cycle, bounding a face $f$, say. Because the $a b$-edges of $\Omega$ are not parallel on $G_{T}$, there are only two possible edge classes, I and II, say, of $c d$-edges on $G_{T}$. We assume that I is the class containing the $c d$-edges of $\Omega$. Since the edges of $\sigma$ cannot lie in a disk in $\widehat{T}$ 


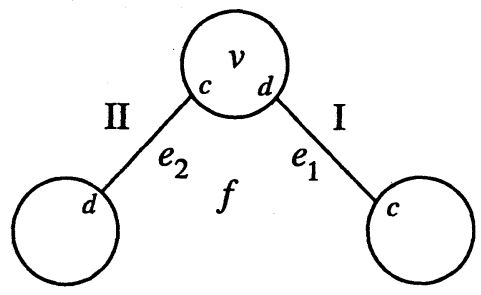

Figure 5.18.

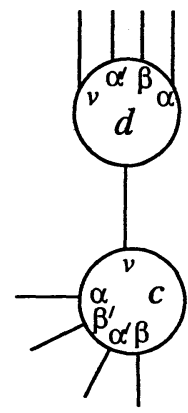

Figure 5.19.

by Lemma 5.5, there must be a corner of $f$ at a vertex $v$ of $G_{Q}$ where an edge $e_{1}$ of $\sigma$ in class I is incident to $v$ with label $d$ and an edge $e_{2}$ of $\sigma$ in class II is incident to $v$ with label $c$. See Figure 5.18.

Thus the label $v$ at vertex $c$ of $G_{T}$ corresponding to $e_{2}$ must lie in $s(c ; \alpha, \beta)$. Then the $\operatorname{arcs}$ of $\partial Q \cap H_{c d}$ force the label $v$ at vertex $d$ corresponding to $e_{1}$ to lie in $s\left(d ; \beta^{\prime}, \alpha^{\prime}\right)$. See Figure 5.19.

But then the labels $\alpha, \alpha^{\prime}$ at vertex $d$ violate Theorem 5.2.

\section{Strong invertibility.}

Recall that a knot $K$ is strongly invertible if there exists an orientationpreserving involution $\tau: S^{3} \rightarrow S^{3}$ such that $\tau(K)=K$ and $\tau \mid K$ is orientation reversing. It follows that the fixed point set of $\tau$ is a circle (which must be unknotted by [Wa]) meeting $K$ in two points.

Eudave-Muñoz has shown [EM1] that if $K$ is a strongly invertible hyperbolic knot such that $K(\gamma)$ contains an incompressible torus for some $\gamma$ with $\Delta(\gamma, \mu)=2$, and if $\widehat{T} \subset K(\gamma)$ is such a torus which minimizes $t=\left|\widehat{T} \cap K_{\gamma}\right|$, then $t=2$. In the present section we show that the existence of certain configurations in $G_{Q}$ implies that $K$ is strongly invertible. Since we are assuming throughout that $t=4$, we conclude that these configurations cannot 


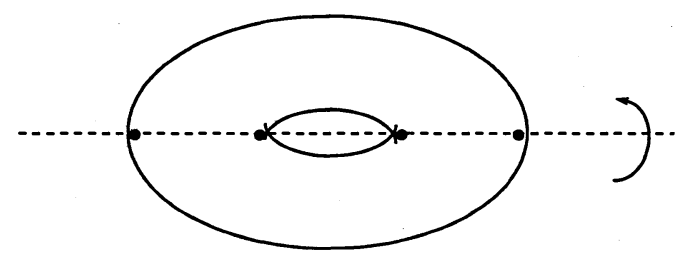

Figure 6.1.

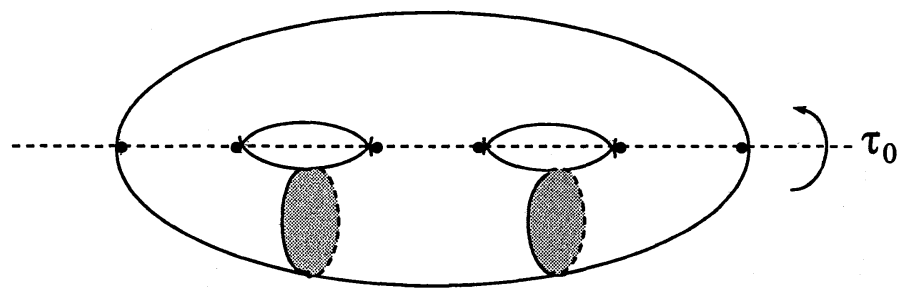

Figure 6.2.

occur.

Lemma 6.1. Let $V$ be a solid torus and let $\tau: \partial V \rightarrow \partial V$ be an involution with exactly four fixed points. Then $\tau$ extends to an involution of $V$.

Proof. An Euler characteristic calculation shows that the quotient $\partial V / \tau$ is a 2-sphere. Then uniqueness of coverings shows that $\tau$ is conjugate to the involution shown in Figure 6.1.

Parametrizing $\partial V$ as $S^{1} \times S^{1}$, this is the map $-I d$. Hence $\tau$ extends over $V$.

Lemma 6.2. Let $W$ be a handlebody of genus 2 and let $\tau: \partial W \rightarrow \partial W$ be an involution with exactly six fixed points. Then $\tau$ extends to an involution of $W$.

Proof. Let $W_{0}$ be a "standard" handlebody of genus 2; see Figure 6.2. Arguing as in the proof of Lemma 6.1, we see that there is a homeomorphism $h: \partial W \rightarrow \partial W_{0}$ such that $\tau=h^{-1} \tau_{0} h$, where $\tau_{0}: \partial W_{0} \rightarrow \partial W_{0}$ is the involution shown in Figure 6.2.

Let $\bar{\tau}_{0}: W_{0} \rightarrow W_{0}$ be the obvious extension of $\tau_{0}$. Let $\bar{g}: W \rightarrow W_{0}$ be a homeomorphism, and let $g: \partial W \rightarrow \partial W_{0}$ be $\bar{g} \mid \partial W$. By [V], $h g^{-1}$ is isotopic to a homeomorphism that commutes with $\tau_{0}$. Hence we may suppose that 

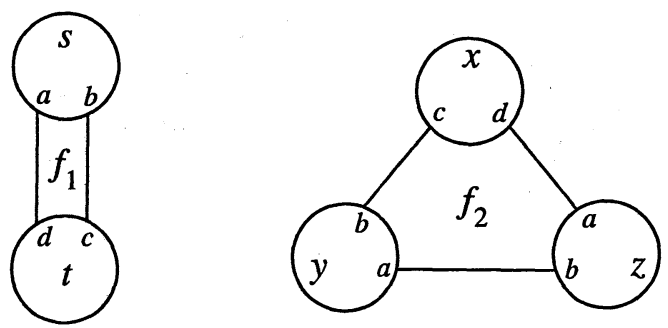

Figure 6.3.

$\bar{g}$ is chosen so that $h g^{-1}$ commutes with $\tau_{0}$. Now define $\bar{\tau}=\bar{g}^{-1} \bar{\tau}_{0} \bar{g}$. Then $\bar{\tau}$ is an involution of $W$ such that $\bar{\tau} \mid \partial W=g^{-1} \tau_{0} g=h^{-1} \tau_{0} h=\tau$.

Theorem 6.3. Suppose that $G_{Q}$ contains a da-Scharlemann cycle of length 2 or 3. Then the faces in Figure 6.3 do not both occur in $G_{Q}$.

To prove Theorem 6.3 we will need Lemmas 6.4 and 6.5 below.

Lemma 6.4. Corresponding edges of $f_{1}$ and $f_{2}$ (i.e., the da-edges or the bc-edges) are not parallel on $G_{T}$.

Proof. Suppose without loss of generality that the $d a$-edges are parallel. Then band summing $f_{1}$ and $f_{2}$ via an arc in $T$ and sliding the resulting disk over $H_{a b}$ and $H_{c d}$ gives rise to a boundary compressing disk for $T$. See Figure 6.4.

Thus, up to homeomorphism, the edges of $f_{1}$ and $f_{2}$ appear on $G_{T}$ as shown in Figure 6.5.

Let $A \subset \widehat{T}$ be an annulus containing the edges of $f_{1}$ and $f_{2}$. Surgering $A$ using the 1-handles $H_{a b}, H_{c d}$ and the 2-handles $N\left(f_{1}\right), N\left(f_{2}\right)$ clearly gives an annulus $B \subset X$. Let $C=\widehat{T}-A$. Then the torus $S=B \cup C$ bounds a solid torus $V \subset X$ (since $X$ contains no incompressible torus, is irreducible, and $C$ is incompressible in $X$ ).

Lemma 6.5. The core of $C$ has algebraic intersection number 1 with the meridian of $V$.

Proof. Assume otherwise.

Let $f$ be the face bounded by the $d a$-Scharlemann cycle $\sigma$ of length 2 or 3. 


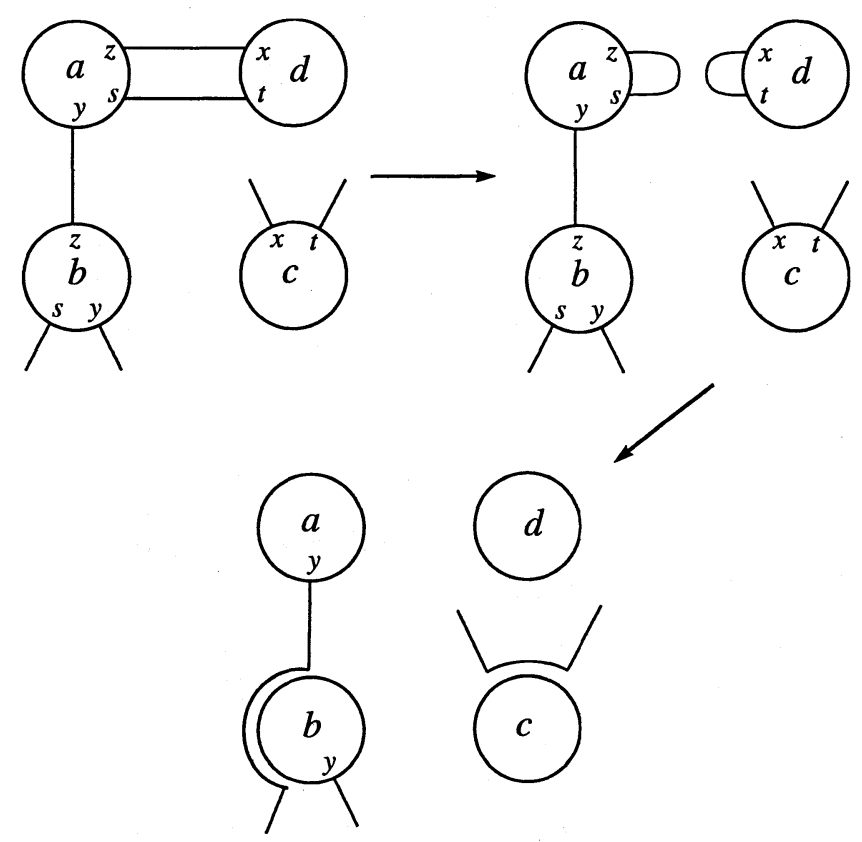

Figure 6.4.

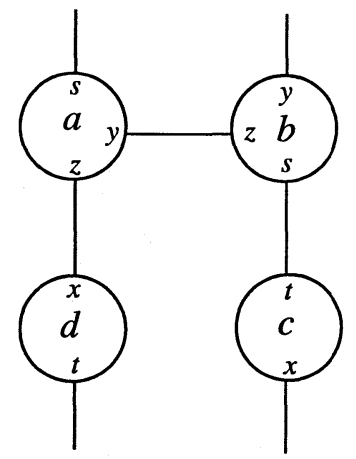

Figure 6.5. 


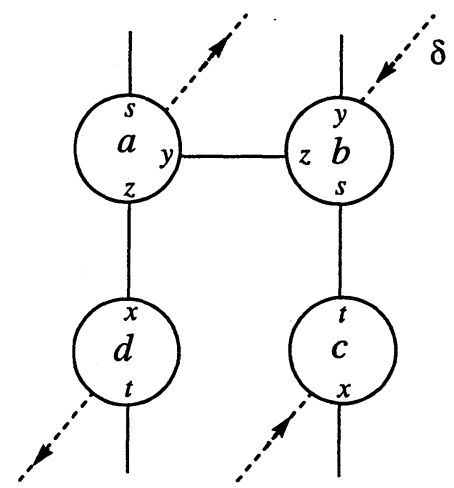

Figure 6.6.

Let $A^{\prime} \subset A$ be an annulus containing the $b c$-edges of $f_{1}$ and $f_{2}$, and disjoint from the edges of $f\left(\right.$ and the vertices $d$ and $a$ ). Let $C^{\prime}=\widehat{T}-A^{\prime}$. By Lemma 3.7 of [GLu1], $V^{\prime}=\operatorname{nhd}\left(C^{\prime} \cup H_{d a} \cup f\right)$ is a solid torus whose meridian intersects the core of $C^{\prime}$ algebraically $n$ times, where $n$ is the length of $\sigma$.

Then $M=V \cup_{C} V^{\prime}$ is a Seifert fiber space over the disk with two exceptional fibers. (Note that the core of $C$ cannot be a meridian of $V$, as this would produce a punctured lens space in $K(\gamma)$.) Since $\left|\partial M \cap K_{\gamma}\right|=2$, $\partial M$ must compress in $K(\gamma)$. Looking at the intersection of a compressing disk with $A^{\prime}$ shows that $A^{\prime}$ is boundary parallel in $K(\gamma)-M$. But this contradicts the incompressibility of $\widehat{T}$.

Since $K(\gamma)$ is irreducible and $\widehat{T}$ is incompressible, it follows that $\partial M$ bounds a solid torus $V^{\prime \prime}$ in $K(\gamma)$. Then $K(\gamma)=V \cup V^{\prime} \cup V^{\prime \prime}$ is a Seifert fiber space over the 2-sphere with at most three exceptional fibers (again using the irreducibility of $K(\gamma)$ ). But since $K(\gamma)$ contains an incompressible torus (and $H_{1}(K(\gamma))$ is finite), this is a contradiction.

Proof of Theorem 6.3. Let $X$ be the side of $T$ containing $\partial H_{a b}$ and $\partial H_{c d}$. Let $\delta \subset \partial X$ be the simple closed curve pictured in Figure 6.6 (note that $\delta$ runs once over each of $\partial H_{a b}, \partial H_{c d}$ ).

Let $\tau^{\prime}: T \rightarrow T$ be the involution indicated in Figure 6.7, with the following properties:

(1) $\tau^{\prime}$ interchanges vertices $d$ and $a$ and vertices $b$ and $c$;

(2) $\tau^{\prime}$ leaves each edge of $f_{1}$ invariant;

$(3)^{\prime} \tau^{\prime}$ interchanges the two $\operatorname{arcs} \delta \cap T$. 


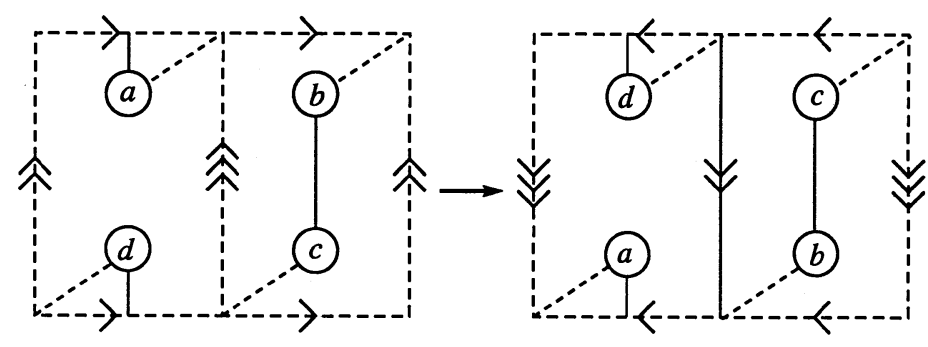

Figure 6.7.

Furthermore, after isotoping the edges of $f$ on $T$ we may assume

(4) $\tau^{\prime}$ leaves the union of the edges of $f$ invariant.

(Note that this isotopy will result in one or two of the edges of $f$ coinciding with one or two of the $d a$-edges of $f_{1}$ and $f_{2}$, according as $\sigma$ has length 2 or 3.)

Since $\delta$ is disjoint from $\partial f_{1}$ and $\partial f_{2}$, we may regard it as lying on $S=$ $B \cup C$. Note that $\delta$ has algebraic intersection number 1 with a core of $C$. Therefore, by Lemma 6.5, by applying to $T$ a suitable power $\varphi$ of a Dehn twist along the core of $C$, we may assume that $\varphi(\delta)$ bounds a meridian disk $D$ of $V$. Define $\tau=\varphi \tau^{\prime} \varphi^{-1}$. Then $\tau$ satisfies (1), (2) and (4) above, and, instead of $(3)^{\prime}$ :

(3) $\tau$ interchanges the two $\operatorname{arcs} \partial D \cap T$.

Claim 6.6. $\tau$ extends to an involution of $X$ with no fixed points on $\partial X-T$.

Proof. First extend $\tau$ to an involution of $\partial X=T \cup \partial H_{a b} \cup H_{c d}$ (interchanging the 1-handles), so that $\partial f_{1}$ and $\partial D$ are invariant. Note that $\tau$ has no fixed points on $\partial D$ and exactly two fixed points on $\partial f_{1}$. We may now extend $\tau$ over $f_{1}$ and $D$, and hence over $N=\operatorname{nhd}_{X}\left(\partial X \cup f_{1} \cup D\right)$. Since $\partial f_{1}$ and $\partial D$ are clearly homologically independent on $\partial X, \partial N=\partial X \cup F$ where $F$ is a torus. For the usual reasons, $F$ bounds a solid torus $V^{\prime} \subset X$. Note that $\tau \mid T$ has exactly four fixed points, as does $\tau \mid \partial X$. Thus $\tau \mid F$ has four fixed points (it loses two after surgering by $f_{1}$ but gains two after surgering by $D$ ). By Lemma 6.1, $\tau \mid F$ extends over $V^{\prime}$. We have thus extended $\tau$ over $X$.

Claim 6.7. $\tau$ extends to an involution of $X^{\prime}$ with exactly four fixed points on $\partial X^{\prime}-T$. 


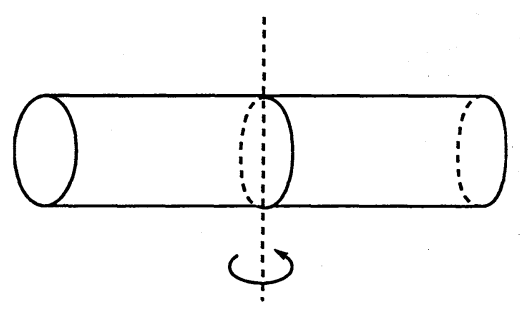

Figure 6.8.

Proof. First extend $\tau$ over $\partial X^{\prime}=T \cup \partial H_{d a} \cup \partial H_{b c}$, by mapping each 1-handle to itself by the involution shown in Figure 6.8.

Then $\partial f$ may be isotoped on $\partial X^{\prime}$ so that it is invariant under $\tau$, and so that $\tau \mid \partial f$ has exactly two fixed points. (If $f$ has two edges, each edge contains a fixed point; if $f$ has three edges, one fixed point occurs on an edge of $f$ and the other on a component of $\partial f \cap \partial H_{d a}$.) Thus $\tau$ may be extended over $f$ and hence over $N^{\prime}=\operatorname{nhd}_{X^{\prime}}\left(\partial X^{\prime} \cup f\right)$. Note that $\partial N^{\prime}=\partial X^{\prime} \cup F$ where $F$ is a surface of genus 2 , and that $\tau \mid F$ has exactly six fixed points.

Subclaim 6.8. $X^{\prime}=N^{\prime} \cup_{F} W$ where $W$ is a handlebody of genus 2 .

Proof. If $F$ were incompressible in $X^{\prime}$ it would be incompressible in $E(K)$ (since $T$ is incompressible). On the other hand there is an annulus in $E(K)$ (in fact, in $X^{\prime}$ ) with one boundary component on $F$ and the other having slope $\gamma$ on $\partial E(K)$. An easy combinatorial argument then shows that $F$ would remain incompressible under the meridional Dehn filling, since $\Delta(\gamma, \mu)=2$ (see for example [CGLS, Theorem 2.4.3]). This contradiction implies that $F$ compresses in $X^{\prime}$. Since $F$ is incompressible in $N^{\prime}$, it must compress in $W$. Since $X^{\prime}$ contains no incompressible torus, and is irreducible, $W$ must be a handlebody.

By Lemma $6.2, \tau \mid F$ can be extended over $W$. Since that, by construction, $\tau \mid \partial X^{\prime}-T$ has exactly four fixed points, this proves Claim 6.7.

Claims 6.6 and 6.7 show that $\tau$ can be extended to an involution of $E(K)$ so that $\tau \mid \partial E(K)$ has exactly four fixed points. By Lemma 6.1, $\tau$ extends to an involution of $S^{3}$, showing that $K$ is strongly invertible. But now [EM1] shows that $t=2$, a contradiction.

Theorem 6.9. Suppose that $G_{Q}$ contains 

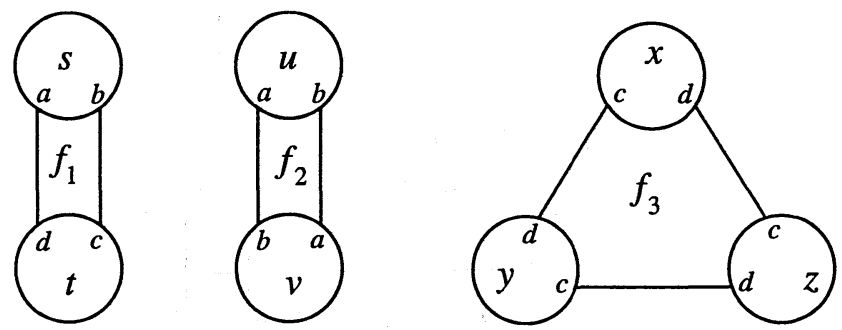

Figure 6.9.

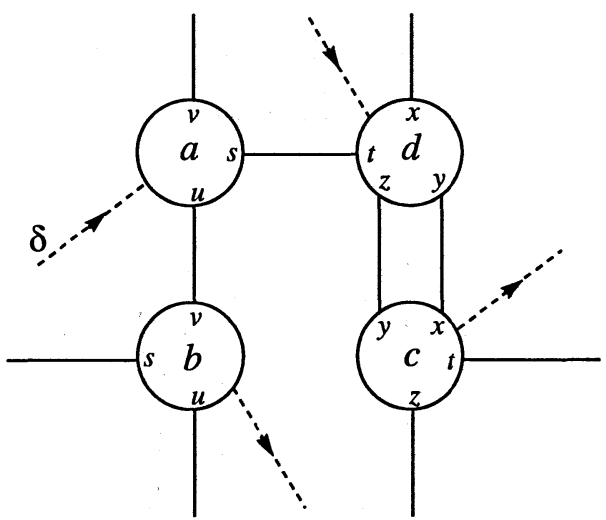

Figure 6.10.

(1) a da-Scharlemann cycle of length 2 or 3 , and

(2) bc-edges which are not parallel on $G_{T}$.

Then $G_{Q}$ does not contain an ab-Scharlemann cycle of length 2 , a cdScharlemann cycle of length 3 , and an $(a b, c d)$-bigon.

Proof. Suppose for contradiction that $G_{Q}$ contains faces $f_{1}, f_{2}, f_{3}$ as shown in Figure 6.9. After a homeomorphism of $T$ we may assume (using Lemmas 5.5 and 5.6) that the edges of $f_{1}, f_{2}$ and $f_{3}$ appear in $G_{T}$ as shown in Figure 6.10.

Let $f$ be the face bounded by the $d a-$ Scharlemann cycle of length 2 or 3. By Lemma 5.6, the edges of $f$ lie in an annulus $A$ in $\widehat{T}$.

Claim 6.10. The core of $A$ is not parallel to the simple closed curve in $\widehat{T}$ defined by the edges of $f_{2}$.

Proof. Assume otherwise. Then there would be an annulus $A$ in $\widehat{T}$ containing 


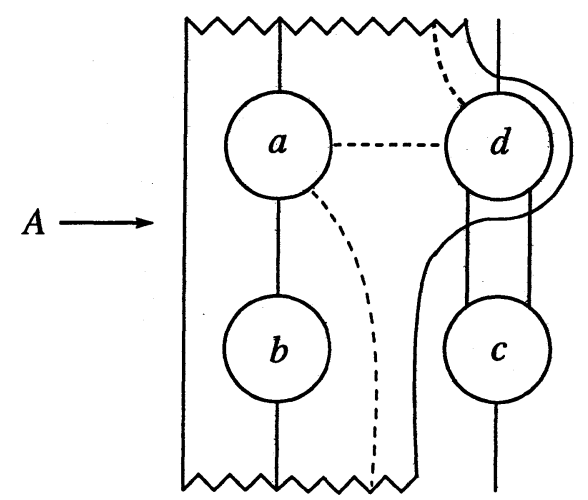

Figure 6.11.

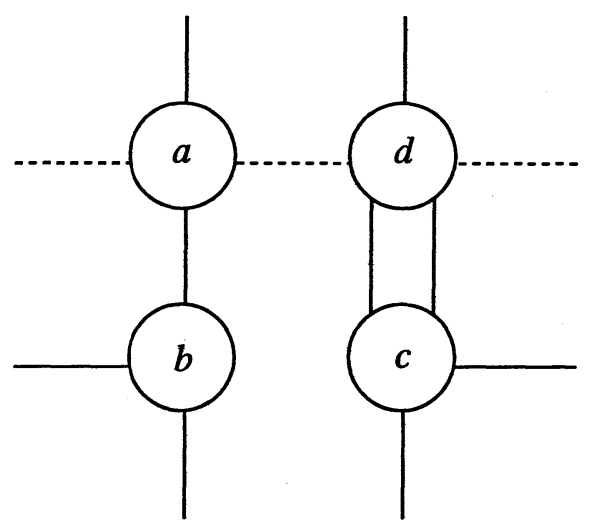

Figure 6.12.

vertices $a, b, d$ and the edges of $f_{2}$ and $f$, and disjoint from vertex $c$. See Figure 6.11.

The argument of Lemma 3.7 of [GLu1] shows that $M=\operatorname{nhd}\left(A \cup H_{d a} \cup\right.$ $\left.H_{a b} \cup f \cup f_{2}\right)$ is a Seifert fiber space over the disk with two exceptional fibers. Since $\left|\partial N \cap K_{\gamma}\right|=2, \partial M$ must be compressible in $K(\gamma)$ by the minimality of $\widehat{T}$. But arguing exactly as in the last paragraph of the proof of Lemma 6.5, this is a contradiction.

Claim 6.10 and the hypothesis that there are non-parallel $b c$-edges on $G_{T}$ imply that the edges of $f$ lie in the edge classes indicated by dotted lines in Figure 6.12.

Let $X$ be the side of $T$ containing $\partial H_{a b}$ and $\partial H_{c d}$. Let $\delta$ be the simple closed curve on $\partial X$ pictured in Figure 6.10. (Note that $\delta$ runs once over 


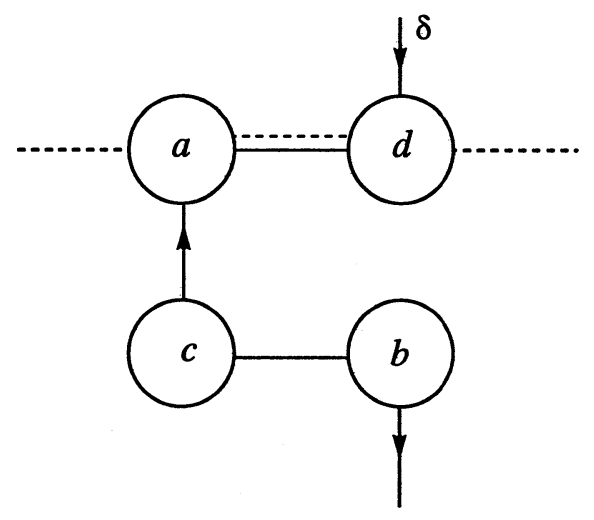

Figure 6.13.

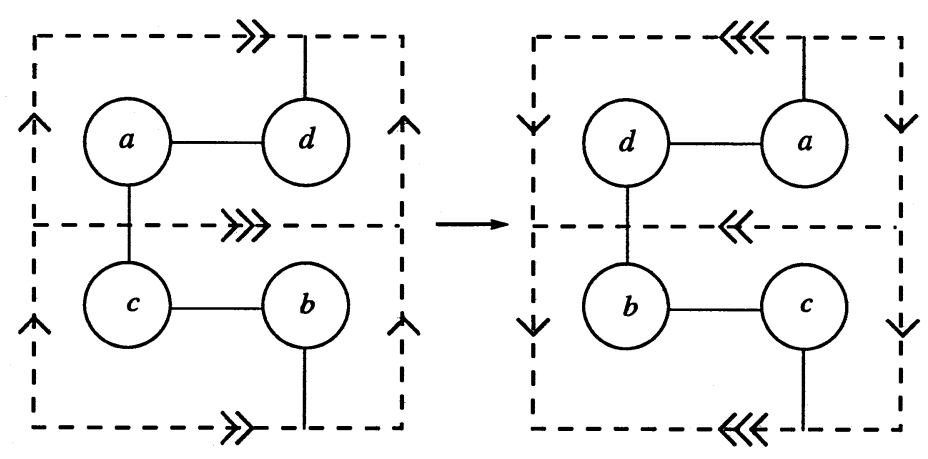

Figure 6.14.

each of $\partial H_{a b}$ and $\partial H_{c d}$.) Since $f_{1}, f_{2}, f_{3}$ are clearly independent, and since $\delta$ is disjoint from $\partial f_{1} \cup \partial f_{2} \cup \partial f_{3}, \delta$ bounds a disk $D$ in $X$.

There is a homeomorphism taking $T$ to Figure 6.13, where only the arcs $\partial f_{1} \cap T, \partial D \cap T$ and the edge classes of $f$ are pictured.

Let $\tau: T \rightarrow T$ be the involution indicated in Figure 6.14.

Note that

(1) $\tau$ interchanges vertices $d$ and $a$ and vertices $b$ and $c$;

(2) $\tau$ leaves each edge of $f_{1}$ invariant;

(3) $\tau$ interchanges the two $\operatorname{arcs} \partial D \cap T$;

and after isotoping the edges of $f$ (which may result in one of these edges coinciding with the $d a$-edge of $f_{1}$ )

(4) $\tau$ leaves the union of the edges of $f$ invariant. 


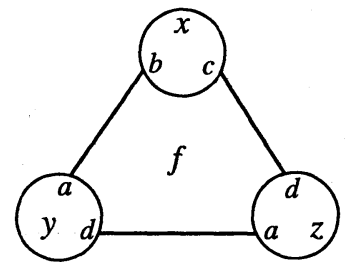

Figure 6.15.

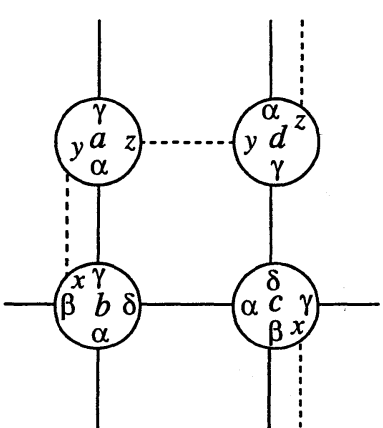

(a)

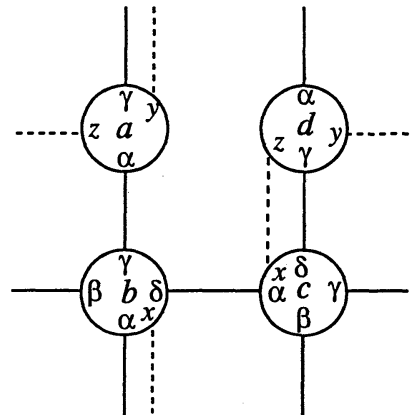

(b)

Figure 6.16.

These are exactly the same properties as those of the $\tau$ in the proof of Theorem 6.3. Hence a verbatim application of the subsequent part of that proof, i.e., starting at Claim 6.6, gives us the desired contradiction.

Theorem 6.11. $G_{Q}$ does not contain both a configuration as in Figure 5.9 and a face as in Figure 6.15.

Proof. Assume for contradiction the existence of both configurations in $G_{Q}$. The argument breaks up into two cases according to whether the edges of Figure 5.9 appear on $G_{T}$ as in Figure 5.12(i) (Case (i)) or 5.12(ii) (Case (ii)). Let $f_{1}, f_{2}, f_{3}$ be the faces of Figure 5.9 and $f$ the face of Figure 6.15.

Case (i). Note that the arcs of $\left(\partial f_{1} \cup \partial f_{2} \cup \partial f\right) \cap H_{d a}$ relate the labelling at vertex $a$ to that at vertex $d$. Similarly the arcs of $\left(\partial f_{1} \cup \partial f_{2} \cup \partial f\right) \cap H_{b c}$ relate the labellings at vertices $b$ and $c$. Using this, the edges of $\partial f$ must lie on $G_{T}$ as shown in Figure 6.16(a) or (b). We assume the configuration is as in (a); the argument for (b) is identical.

Let $\tau: T \rightarrow T$ be the involution indicated in Figure 6.17. 


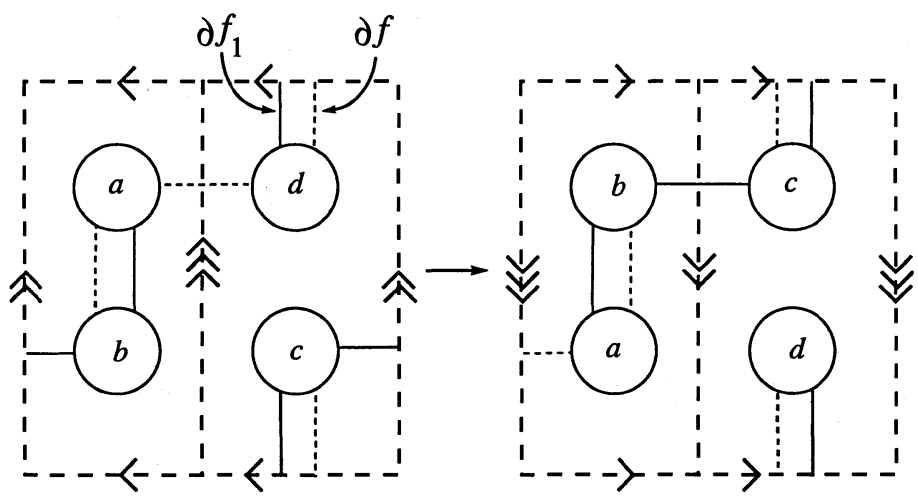

Figure 6.17.

Suppose that $f_{1}, f_{2}, f$ lie in $X$, and $f_{3}$ in $X^{\prime}$.

Claim 6.12. $\tau$ extends to an involution of $X$ with no fixed points on $\partial X-T$.

Proof. First extend $\tau$ to an involution of $\partial X$ by having $\tau$ interchange $\partial H_{d a}$ and $\partial H_{b c}$. Then $\partial f_{1}$ and $\partial f$ may be isotoped so that $\tau$ interchanges them. Thus $\tau$ may be extended over $f_{1}$ and $f$, and hence over $N=\operatorname{nhd}_{X}(\partial X \cup$ $\left.f_{1} \cup f\right)$. We have $\partial N=\partial X \cup F$, where $F$ is a torus and $\tau \mid F$ is an involution with exactly four fixed points. As before, $F$ bounds a solid torus $V$ in $X$ and $\tau$ extends over $V$ by Lemma 6.1.

Claim 6.13. $\tau$ extends to an involution of $X^{\prime}$ with exactly four fixed points on $\partial X^{\prime}-T$.

Proof. This is exactly like the proof of Claim 6.7, replacing the $f$ of that argument by $f_{3}$.

Claims 6.12 and 6.13 give the desired contradiction, exactly as in the proof of Theorem 6.3.

Case (ii). As in Case (i) the arcs of $\left(\partial f_{1} \cup \partial f_{2} \cup \partial f\right) \cap\left(\partial H_{d a} \cup \partial H_{b c}\right)$ force the edges of $\partial f$ to lie on $G_{T}$ as pictured in Figure 6.18(a) or (b).

We assume the configuration is as in Figure 6.18(a). The argument for (b) is similar: after applying a Dehn twist to $T$ along the central vertical curve we get (the reflection of) configuration (a) (with $f_{1}$ and $f_{2}$ interchanged). 


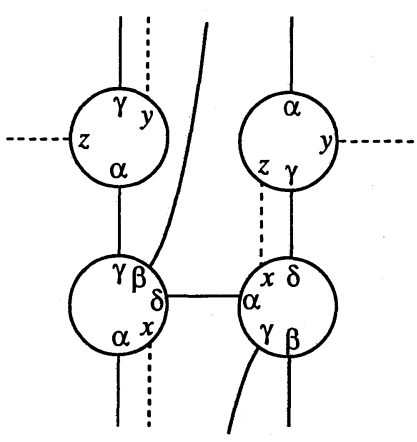

(a)

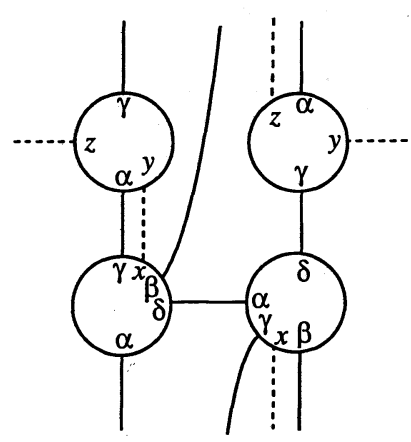

(b)

Figure 6.18.

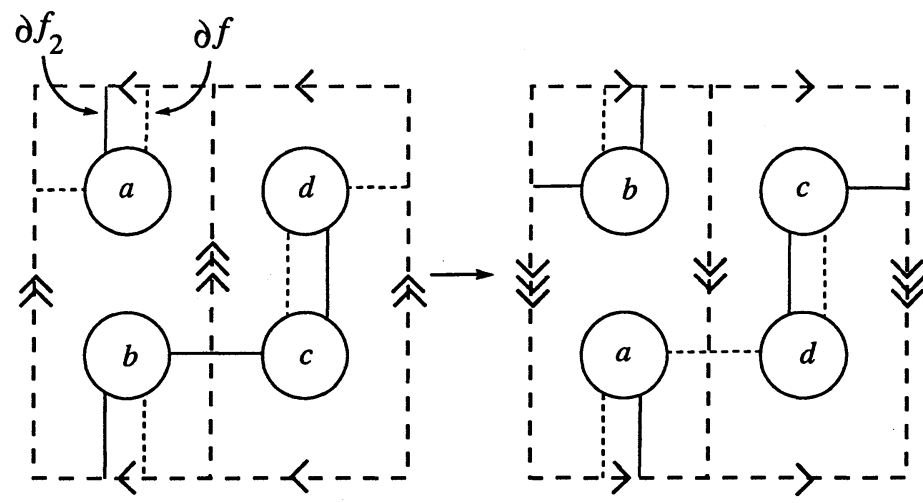

Figure 6.19.

Let $\tau$ be the involution pictured in Figure 6.19.

Claims 6.12 and 6.13 now hold for $\tau$, as in Case (i). (The argument for Claim 6.13 is exactly the same; for Claim 6.12 we here use $f_{2}$ instead of $f_{1}$.) Thus again we get a contradiction.

This completes the proof of Theorem 6.11.

Theorem 6.14. Suppose that $G_{Q}$ contains a cd-Scharlemann cycle. Then $G_{Q}$ does not contain a configuration as in Figure 6.20.

First we have the following lemma.

Lemma 6.15. Suppose that $G_{Q}$ contains a configuration $\Omega$ as in Figure 6.20. Then the cd-edges of $\Omega$ are not parallel on $G_{T}$. 


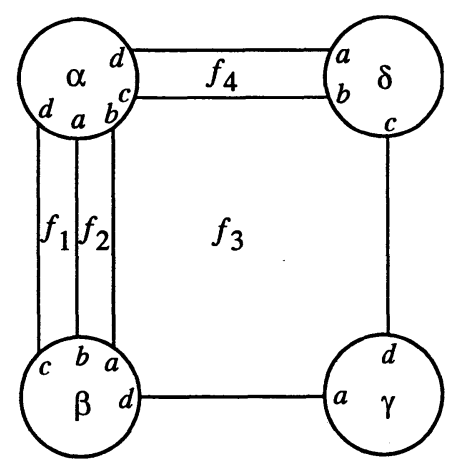

Figure 6.20.

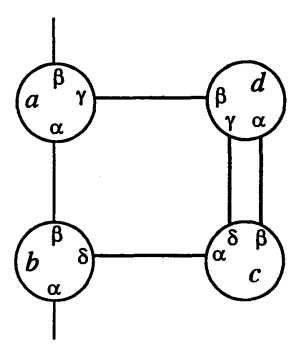

(a)

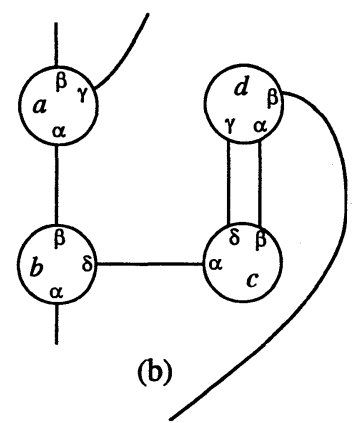

Figure 6.21.

Proof. Let $f_{i}, i=1,2,3,4$, be the faces of $\Omega$ pictured in Figure 6.20.

Assume for contradiction that the $c d$-edges of $\Omega$ are parallel on $G_{T}$. By Lemma 5.5, the $a b$-edges of $\Omega$ are not parallel on $G_{T}$. Then, up to homeomorphism of $T$, the edges of $f_{1}$ and $f_{3}$ appear on $G_{T}$ as shown in Figure 6.21(a) or (b).

In Case (a), let $A$ be an annulus in $\widehat{T}$ containing the edges of $f_{1}$ and $f_{3}$. Let $M=\operatorname{nhd}\left(A \cup H_{d a} \cup H_{b c} \cup f_{1} \cup f_{3}\right)$. Then $\pi_{1}(M) \cong\left\langle x, y, z \mid x y, z y^{2} x^{2}\right\rangle$, where $x, y, z$ are represented by the cores of $H_{d a}, H_{b c}$ and $A$ respectively. Thus $z=1$ in $\pi_{1}(M)$. But this contradicts the incompressibility of $\widehat{T}$ in $K(\gamma)$.

In Case (b), a homeomorphism of $T$ takes Figure 6.20(b) to Figure 6.22.

We may now apply the argument of Case (a) to again contradict the incompressibility of $\widehat{T}$.

Proof of Theorem 6.14. Assume otherwise. By Lemma 6.15, and by con- 


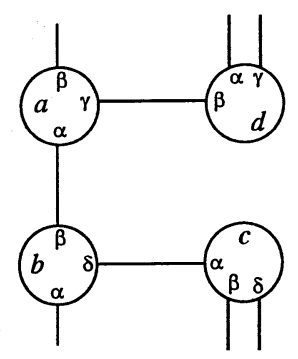

Figure 6.22.
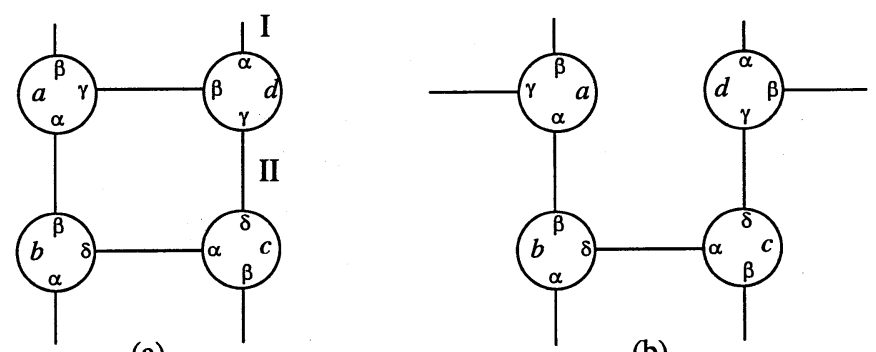

(a)

(b)

Figure 6.23.

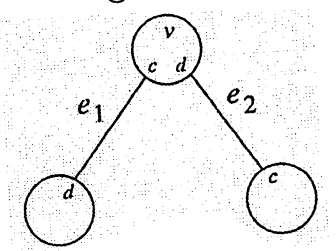

Figure 6.24.

sidering the arcs $\left(\partial f_{1} \cup \partial f_{3}\right) \cap H_{d a}$ and $\left(\partial f_{1} \cup \partial f_{3}\right) \cap H_{b c}$, one sees that the edges of $f_{1}$ and $f_{3}$ must appear on $G_{T}$ as in Figure 6.23(a) or (b).

We will treat these two cases separately.

Case (a). Because of the existence of non-parallel ab-edges on $G_{T}, G_{T}$ contains exactly two $c d$-edge classes, I and II, shown in Figure 6.23(a).

Let $\sigma$ be the $c d$-Scharlemann cycle in the hypothesis of the theorem, bounding a face $f$, say, of $G_{Q}$. By Lemma 5.5, $\sigma$ contains an edge $e_{2}$ in class II. Let $v$ be the vertex of $G_{Q}$ at which $e_{2}$ has label $d$, and let $e_{1}$ be the edge of $\sigma$ with label $c$ at $v$. See Figure 6.24.

Using the arcs of $\left(\partial f_{2} \cup \partial f_{4}\right) \cap H_{a b}$, we see that the $d a$-edge of $f_{4}$ must appear on $G_{T}$ as in Figure 6.25.

Since $e_{2}$ is in class II, $v \in s\left(d ; \alpha^{\prime}, \beta\right)$. Also, we must have $\beta^{\prime} \in s\left(d ; \alpha, \alpha^{\prime}\right)$. 


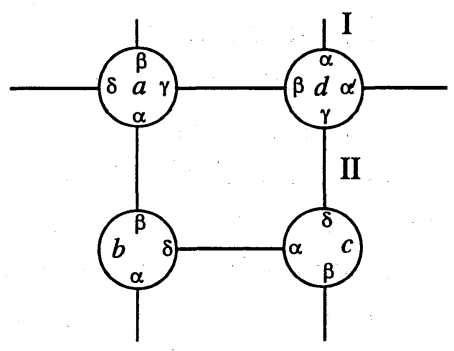

Figure 6.25.

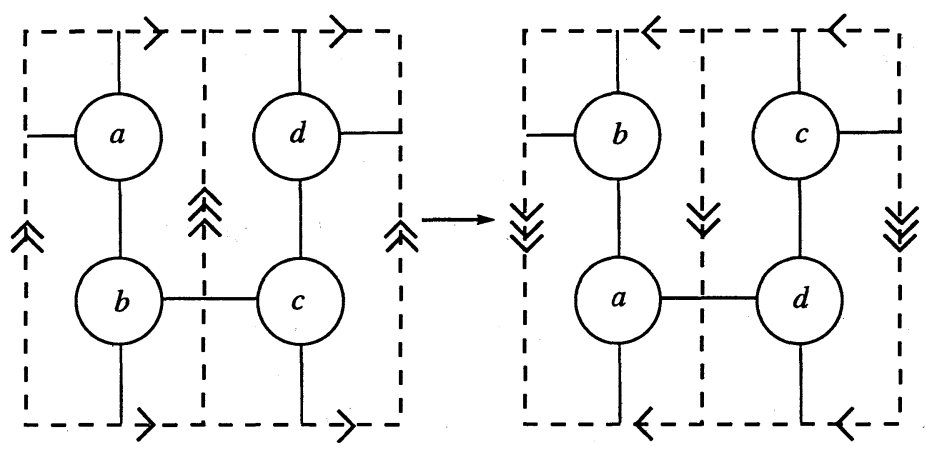

Figure 6.26.

Hence $v \in s\left(d ; \alpha^{\prime}, \beta^{\prime}\right)$. The arcs of $\left(\partial f \cup \partial f_{4} \cup \partial(\operatorname{vertex} \beta)\right) \cap H_{c d}$ now dictate that $v \in s(c ; \beta, \alpha)$. Hence the edge $e_{1}$ is in class I. Furthermore, $\beta \in$ $s\left(d ; v, \beta^{\prime}\right)$, and therefore (by considering the $\operatorname{arcs}$ of $\left.\partial Q \cap H_{c d}\right) \beta^{\prime} \in s(c ; \beta, v)$. This implies that both edges of $G_{T}$ with label $\beta$ at $c$ are $c d$-edges in class I. But this contradicts Theorem 5.2.

Case (b). Let $\tau$ be the involution of $T$ indicated in Figure 6.26.

Note that

(1) $\tau$ interchanges vertices $a$ and $b$ and vertices $c$ and $d$;

(2) $\tau$ leaves the union of the edges of $f_{1}$ invariant;

(3) $\tau$ leaves the union of the edges of $f_{3}$ invariant.

Suppose $f_{1}, f_{3}$ lie in $X$, and $f_{2}$ in $X^{\prime}$.

Claim 6.16. $\tau$ extends to an involution of $X$ with no fixed points on $\partial X-T$. 
Proof. Extend $\tau$ over $\partial X$, by interchanging $\partial H_{d a}$ and $\partial H_{b c}$. Then $\partial f_{1}$, $\partial f_{3}$ may be isotoped on $\partial X$ so that each is invariant under $\tau$, with $\tau \mid \partial f_{1}$, $\tau \mid \partial f_{3}$ each having two fixed points. Thus $\tau$ may be extended over $N=$ $\operatorname{nhd}\left(\partial X \cup f_{1} \cup f_{3}\right)$. Note that $\partial N=\partial X \cup F$, where $F$ is a torus which, as usual, bounds a solid torus $V$ in $X$, and that $\tau \mid F$ has no fixed points. Thus $\tau \mid F$ is a 2-fold covering transformation, with quotient a torus (since $\tau \mid F$ is orientation preserving). Hence $F$ can be parametrized as $S^{1} \times S^{1}$ so that $\tau \mid F$ is given by $(\theta, \varphi) \mapsto(\theta, \varphi+\pi)$. It follows easily that $\tau \mid F$ extends to an involution of $V$.

Claim 6.17. $\tau$ extends to an involution of $X^{\prime}$ with exactly four fixed points on $\partial X^{\prime}-T$.

Proof. This is exactly like the proof of Claim 6.7, here using the face $f_{2}$.

Claims 6.16 and 6.17 now give a contradiction, as in proof of Theorem 6.3.

We have thus shown that neither Case (a) nor Case (b) can hold, proving the theorem.

\section{Euler characteristic arguments.}

In this section we apply Euler characteristic arguments to the graphs $\Lambda_{x}$ and $\Lambda$ to get a lower bound on the number of faces of $\Lambda_{x}$ of length at most 4 (Theorem 7.1), and to show that $\Lambda$ must contain a vertex at which one of a certain number of combinations of faces of length at most 4 must be incident (Theorem 7.2).

Theorem 7.1. For any label $x$ of $G_{Q}$,

$$
3 n_{2}^{x}+2 n_{3}^{x}+n_{4}^{x}>V,
$$

where $n_{i}^{x}$ denotes the number of faces of $\Lambda_{x}$ of length $i$, and $V$ is the number of vertices of $\Lambda$.

Proof. Fix $x$ and write $n_{i}=n_{i}^{x}$.

The graph $\Lambda$ is contained in the disk $D_{\Lambda}$ in $\widehat{Q}$ bounded by $\Lambda$ (see [GLu1]). Let $\alpha=\alpha_{x}$ be the number of ghost $x$-labels. By Theorem 5.6 of [GLu1], $\alpha \leq 2$. Let $\omega$ be the number of edges (counted with multiplicity) of the 
outside region of $\Lambda_{x}$ (i.e., the region containing $\partial D_{\Lambda}$ ). Let $V, E, F$ be the number of vertices, edges and faces of $\Lambda_{x}$. Then

$$
\sum_{j} j n_{j}+\omega=2 E
$$

In particular,

$$
5 F-n_{4}-2 n_{3}-3 n_{2}+\omega \leq 2 E .
$$

Hence

$$
F \leq \frac{2}{5} E+\frac{1}{5} n_{4}+\frac{2}{5} n_{3}+\frac{3}{5} n_{2}-\frac{1}{5} \omega .
$$

This, along with $2 V=E+\alpha$ (use the parity rule) gives

$$
1 \leq V-E+F \leq\left(\frac{E}{2}+\frac{\alpha}{2}\right)-E+\left(\frac{2}{5} E+\frac{1}{5} n_{4}+\frac{2}{5} n_{3}+\frac{3}{5} n_{2}-\frac{1}{5} \omega\right) .
$$

That is,

$$
\begin{aligned}
3 n_{2}+2 n_{3}+n_{4} & \geq \frac{E}{2}-\frac{5}{2} \alpha+5+\omega \\
& \geq V-3 \alpha+5+\omega .
\end{aligned}
$$

Since $\alpha \leq 2$, if $\omega \geq 2$ we are done. If $\omega \leq 1$, then $\Lambda_{x}$ can have at most one ghost label $x$, i.e., $\alpha \leq 1$, and again we are done.

Regard $\Lambda$ as a graph in the 2-sphere $\widehat{Q}$. Thus $\Lambda$ has one outside face, i.e., the one containing $\partial D_{\Lambda}$; all others are ordinary faces. For each vertex $v$ of $\Lambda$, let $\varphi_{i}(v)$ be the number of ordinary faces of $\Lambda$ of length $i$ incident to $v$ (counted with multiplicity). We will consider faces of order $i, 2 \leq i \leq 4$ (recall that $\varphi_{1}(v)=0$ for all $v$ ). Note that $\sum_{i} \varphi_{i}(v) \leq 8$ for all $v$.

Let $\rho=\left(\rho_{2}, \rho_{3}, \rho_{4}\right)$ be an ordered triple of non-negative integers with $\sum \rho_{i} \leq 8$. We say that $\rho$ is of type $\left[k_{2}, \ldots, k_{m}\right], 2 \leq m \leq 4, k_{m}>0$, if

$$
\begin{aligned}
\rho_{i} & =k_{i}, \quad 2 \leq i \leq m-1, \\
\rho_{m} & \geq k_{m} .
\end{aligned}
$$

We say that $\rho$ is special if it is of one of the following types: [5], [4,2], [4,1,2], $[3,4]$.

We say that a vertex $v$ of $\Lambda$ is of type $\left[k_{2}, \ldots, k_{m}\right]$ if the triple $\varphi(v)=$ $\left(\varphi_{2}(v), \varphi_{3}(v), \varphi_{4}(v)\right)$ is, and similarly we say $v$ is special if $\varphi(v)$ is. (Thus, for example, a vertex of type $[4,1,2]$ has incident to it 4 bigons, 13 -gon, and at least 2 4-gons.) 
Theorem 7.2. $\Lambda$ contains a special vertex.

Corollary 7.3. $\Lambda$ contains a vertex at which at least three bigons of $\Lambda$ are incident.

We shall need the following lemma. For $\rho=\left(\rho_{2}, \rho_{3}, \rho_{4}\right)$ as above define $\alpha(\rho)=\frac{3 \rho_{2}}{2}+\frac{2 \rho_{3}}{3}+\frac{\rho_{4}}{4}$.

Lemma 7.4. If $\alpha(\rho)>7$ then $\rho$ is special.

Proof. Assume $\alpha(\rho)>7$. We enumerate several cases.

(1) $\rho_{2} \geq 5$. Then $\rho$ is of type [5].

(2) $\rho_{2}=4$. Then $\alpha(\rho)>7$ implies $\frac{2 \rho_{3}}{3}+\frac{\rho_{4}}{4}>1$.

If $\rho_{3} \geq 2$, then $\rho$ is of type $[4,2]$.

If $\rho_{3}=1$, then $\frac{\rho_{4}}{4}>\frac{1}{3}$, hence $\rho_{4} \geq 2$, and $\rho$ is of type $[4,1,2]$.

If $\rho_{3}=0$, then $\rho_{4} \leq 4$ (since $\left.\sum \rho_{i} \leq 8\right)$, hence $\frac{\rho_{4}}{4} \leq 1$, a contradiction.

(3) $\rho_{2}=3$. Then $\alpha(\rho)>7$ implies $\frac{2 \rho_{3}}{3}+\frac{\rho_{4}}{4}>\frac{5}{2}$.

If $\rho_{3} \geq 4$, then $\rho$ is of type $[3,4]$.

If $\rho_{3} \leq 3$, then, since $\rho_{3}+\rho_{4} \leq 5$, we have $\frac{2 \rho_{3}}{3}+\frac{\rho_{4}}{4} \leq \frac{2 \cdot 3}{3}+\frac{2}{4}=\frac{5}{2}$, a contradiction.

(4) $\rho_{2}=2$. Then, since $\rho_{3}+\rho_{4} \leq 6$, we have $\alpha(\rho) \leq \frac{3 \cdot 2}{2}+\frac{2 \cdot 6}{3}=7$, a contradiction.

(5) $\rho_{2}=1$. Then $\rho_{3}+\rho_{4} \leq 7$ and $\alpha(\rho) \leq \frac{3}{2}+\frac{2}{3} \cdot 7<7$, a contradiction. $\square$

Proof of Theorem 7.2. Let $V, E, F, \ell$ be the number of vertices, edges, faces, and ghost labels of $\Lambda$, respectively. Then

$$
2 E=8 V-\ell
$$

hence

$$
E=4 V-\frac{\ell}{2}
$$

Also

$$
2=V-E+F
$$


giving

$$
F=3 V+\left(2-\frac{\ell}{2}\right)
$$

Let $F_{i}$ be the number of faces of $\Lambda$ of length $i, i=1,2, \ldots$ Then $\sum_{i} F_{i}=F$, hence

$$
\sum_{i} 5 F_{i}=15 V+\left(10-\frac{5 \ell}{2}\right)
$$

Also,

$$
\sum_{i} i F_{i}=2 E=8 V-\ell
$$

Subtracting, we obtain

$$
4 F_{1}+3 F_{2}+2 F_{3}+F_{4} \geq 7 V+\left(10-\frac{3 \ell}{2}\right) .
$$

Let $\bar{F}_{i}$ be the number of ordinary faces of $\Lambda$ of length $i$. Recall that $\ell \leq 4$. Then, unless the outside face of $\Lambda$ has length 1 and $\ell=4,(7.1)$ implies

$$
3 \bar{F}_{2}+2 \bar{F}_{3}+\bar{F}_{4}>7 \mathrm{~V} .
$$

Now $i \bar{F}_{i}=\sum_{v} \varphi_{i}(v)$, for all $i$. Hence

$$
3 \bar{F}_{2}+2 \bar{F}_{3}+\bar{F}_{4}=\sum_{v}\left(\frac{3 \varphi_{2}(v)}{2}+\frac{2 \varphi_{3}(v)}{3}+\frac{\varphi_{4}(v)}{4}\right)=\sum_{v} \alpha(\varphi(v)) .
$$

Therefore there exists a vertex $v$ of $\Lambda$ such that $\alpha(\varphi(v))>7$. This vertex is special by Lemma 7.4.

Finally, suppose that the outside face of $\Lambda$ has length 1 and $\ell=4$. Then (7.1) gives

$$
\begin{aligned}
3 F_{2}+2 F_{3}+F_{4} & \geq 7 V, \\
\text { i.e., } \quad \sum_{v} \alpha(\varphi(v)) & \geq 7 V .
\end{aligned}
$$

Let $v_{0}$ be the vertex of $\Lambda$ belonging to the outside face. Then, since $\ell=4$, there are only three ordinary faces of $\Lambda$ incident to $v_{0}$ (see Figure 7.1). Hence $\alpha\left(\varphi\left(v_{0}\right)\right) \leq \frac{3 \cdot 3}{2}<7$. It follows that there exists a vertex $v$ with $\alpha(\varphi(v))>7$ in this case also. 


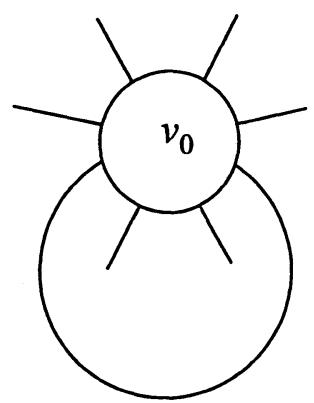

Figure 7.1.

\section{References.}

[CGLS] M. Culler, C. McA. Gordon, J. Luecke, and P.B. Shalen, Dehn surgery on knots, Ann. Math. 125 (1987), 237-300.

[EM1] M. Eudave-Muñoz, Essential tori obtained by surgery on a knot, Pacific J. Math. 167 (1995), 81-116.

[EM2] M. Eudave-Muñoz, Non-hyperbolic manifolds obtained by Dehn surgery on hyperbolic knots, in Geometric Topology, 1993, Georgia International Topology Conference Proceedings, W.H. Kazez, editor, AMS/IP Studies in Advanced Mathematics, Volume 2 (1997), Part 1, 35-61.

[GLi] C. McA. Gordon, R.A. Litherland, Incompressible planar surfaces in 3manifolds, Topology and its Applications, 18 (1984), 121-144.

[GLu1] C. McA. Gordon, J. Luecke, Dehn surgeries on knots creating essential tori, I, Communications in Analysis and Geometry, 3 (1995), 597-644.

[GLu2] C. McA. Gordon, J. Luecke, Only integral Dehn surgeries can yield reducible manifolds, Math. Proc. Camb. Phil. Soc. 102 (1987), 94-101.

[V] O. Ja. Viro, Linkings, 2-sheeted branched coverings, and braids, Mat. Sb. (N.S.) 87 (1972), 216-228. English translation: Math. USSR-Sb. 16 (1972), 223-236.

[Wa] F. Waldhausen, Über involutionen der 3-Sphäre, Topology, 8 (1969), 8191.

RECEIVED JULY 30, 1996. 
Department of Mathematics

The University of TeXas at Austin

AUstin, TX 78712-1082 